\title{
Claudin-1-Dependent Destabilization of the Blood-Brain Barrier in Chronic Stroke
}

\author{
Nikola Sladojevic, ${ }^{1}$ Svetlana M. Stamatovic, ${ }^{1}$ Allison M. Johnson, ${ }^{1}$ Jennifer Choi, ${ }^{1}$ Anna Hu, ${ }^{1}$ Sophie Dithmer, ${ }^{4}$ \\ Ingolf E. Blasig, ${ }^{\circledR}$ Richard F. Keep, ${ }^{2,3}$ and ${ }^{\circledR}$ Anuska V. Andjelkovic ${ }^{1,2}$ \\ ${ }^{1}$ Department of Pathology, ${ }^{2}$ Department of Neurosurgery, ${ }^{3}$ Department of Molecular and Integrative Physiology, University of Michigan Medical School, \\ Ann Arbor MI 48109, and ${ }^{4}$ Leibniz-Institut für Molekulare Pharmakologie, Berlin 13125, Germany
}

Recent evidence suggests that blood-brain barrier (BBB) recovery and reestablishment of BBB impermeability after stroke is incomplete. This could influence stroke recovery, increase the risk of repeat stroke, and be a solid substrate for developing vascular dementia. Although accumulating evidence has defined morphological alterations and underlying mechanisms of tight junction (TJ) changes during BBB breakdown in acute stroke, very little is known about the type of alterations and mechanisms in BBB "leakage" found subacutely or chronically. The current study examined BBB structural alterations during the "BBB leakage" associated with the chronic phase of stroke in male mice and both genders of humans. We found significant upregulation of claudin-1 mRNA and protein, a nonspecific claudin for blood vessels, and downregulation in claudin-5 expression. Morphological and biochemical as well as fluorescence resonance energy transfer and fluorescence recovery after photobleaching analysis of postischemic brain endothelial cells and cells overexpressing claudin-1 indicated that newly synthesized claudin-1 was present on the cell membrane ( $\sim 45 \%)$, was incorporated into the TJ complex with established interaction with zonula occludens-1 (ZO-1), and was building homophilic cis-and trans-interactions. The appearance of claudin- 1 in the TJ complex reduced claudin-5 strands (homophilic claudin-5 cis-and trans-interactions) and claudin-5/ Z0-1 interaction affecting claudin-5 incorporation into the TJ complex. Moreover, claudin-1 induction was associated with an endothelial proinflammatory phenotype. Targeting claudin-1 with a specific C1C2 peptide improved brain endothelial barrier permeability and functional recovery in chronic stroke condition. This study highlights a potential "defect" in postischemic barrier formation that may underlie prolonged vessel leakiness.

Key words: BBB recovery; cerebrovascular injury; claudin-1; stroke

\section{Significance Statement}

Although rarely expressed at the normal blood- brain barrier (BBB), claudin-1 is expressed in pathological conditions. Analyzing poststroke human and mouse blood microvessels we have identified that claudin-1 is highly expressed in leaky brain microvessels. Our results reveal that claudin-1 is incorporated in BBB tight junction complex, impeding BBB recovery and causing BBB leakiness during poststroke recovery. Targeting claudin-1 with a claudin-1 peptide improves brain endothelial barrier permeability and consequently functional neurological recovery after stroke.

\section{Introduction}

Stroke is defined as an abrupt onset of focal or global neurological symptoms caused by ischemia or hemorrhage. Cessation of blood

Received June 6, 2018; revised 0ct. 16, 2018; accepted Nov. 9, 2018.

Author contributions: N.S., S.M.S., I.E.B., and R.F.K. edited the paper; A.V.A. wrote the first draft of the paper. R.F.K. and A.V.A. designed research; N.S., S.M.S., A.M.J., J.C., A.H., and A.V.A. performed research; S.D. and I.E.B. contributed unpublished reagents/analytic tools; N.S., S.M.S., A.M.J., J.C., A.H., and A.V.A. analyzed data; N.S., R.F.K., and A.V.A. wrote the paper.

This work was supported by the National Institutes of Health (National Institute of Neurological Disorders and Stroke Public Health Service Grants NS062853 and NS098066 to A.V.A. and Grant NS098211 to S.M.S. and National Institute on Aging Grant RFA057928 to A.V.A.) and the American Diabetes Association (Grant 1-16-IBS-008 to S.M.S.). Tissue specimens were obtained from the Human Brain and Spinal Fluid Resource Center (West Los Angeles Healthcare Center, Los Angeles, (A) and Mount Sinai NIH Brain Tissue Repository (New York, NY). We thank the Single Molecule Analysis in Real-Time (SMART) Center of the University of Michigan seeded by National Science flow leads to a complex cascade of pathophysiological events at the blood-vascular-parenchymal interface that evolves over time and space and results in damage to neural cells and edema formation (Dirnagl et al., 1999; Fisher and Schaebitz, 2000). The phases after stroke are characterized as hyperacute $(\sim 6 \mathrm{~h}$ from

Foundation MRI-R2-ID Grant DBI-0959823 to Nils G. Walter and J. Damon Hoff for training, technical advice and use of the Olympus STORM-capable microscope. Histology slide scanning was done at the Department of Pathology Core Flow Cytometry Laboratory and Slide Scanning Services.

The authors declare no competing financial interests.

N. Sladojevic's present address: Department of Medicine, Section of Cardiology, University of Chicago, Chicago IL, 60637.

Correspondence should be addressed to Anuska V. Andjelkovic at anuskaa@med.umich.edu.

https://doi.org/10.1523/JNEUROSCl.1432-18.2018

Copyright $\odot 2019$ the authors $\quad 0270-6474 / 19 / 390743-15 \$ 15.00 / 0$ 
symptom onset), acute (6-24 h), subacute ( $24 \mathrm{~h}$ to 6 weeks), and chronic ( $>6$ weeks). During this time, cerebral ischemia evokes a profound and deleterious upregulation in inflammation and triggers multiple cell death pathways, but it also induces regenerative responses, including vascular remodeling, angiogenesis, and neurogenesis (Dirnagl et al., 1999; Chopp et al., 2007). Stroke survivors usually recover at least some function within 3 months, although only $25 \%$ return to prestroke levels (Duncan et al., 2000). One problem regarding stroke recovery is persistent cerebrovascular injury in the area affected by focal infarction. This injury is often associated with increased susceptibility to oxidantinduced stress, aberrant cytokines, and extracellular matrix production and deficient immune responses, affecting endothelial repair and regeneration processes and leading to abnormal barrier function (Wong and Crack, 2008; Deddens et al., 2012). Long-lasting consequences are increased risk of another stroke and a basis for developing poststroke cognitive impairment and vascular dementia (Taheri et al., 2011).

Poststroke cerebrovascular injury predominantly occurs in small caliber vessels, including capillaries, and is associated with BBB dysfunction (Durukan et al., 2009; Kaur et al., 2011). The $\mathrm{BBB}$ is formed by an interdependent network of brain capillary endothelial cells endowed with barrier properties and perivascular cells, astrocytes, and pericytes, which are responsible for barrier induction and maintenance (Hawkins and Davis, 2005; Abbott et al., 2010). A primary BBB property is paracellular permeability regulation due to the presence of tight junctions (TJs) between endothelial cells. The TJ complex involves intricate interactions between transmembrane proteins (claudin-5, occludin, JAM-A), which are important for paracellular space occlusion, and scaffolding proteins [e.g., zonula occludens-1 (ZO-1), ZO-2, VASP] and the actin cytoskeleton, which are vital for physical support and TJ function (Tietz and Engelhardt, 2015; Stamatovic et al., 2016). Transcellular interactions of claudin-5 play the major role in occluding the paracellular space (Nitta et al., 2003; Ohtsuki et al., 2007). Any loosening of its adhesive interactions increases paracellular permeability.

Clinical (MRI) and experimental studies indicate persistent BBB dysfunction with increased paracellular barrier permeability after stroke, questioning the level of BBB recovery (Strbian et al., 2008; Abo-Ramadan et al., 2009; Kaur et al., 2011). BBB hyperpermeability in the subacute and chronic phases after stroke is significantly less than acute changes when there can be uncontrolled and robust extravasation of plasma proteins, leukocyte extravasation, and vasogenic edema (Strbian et al., 2008). However, persistent small BBB leaks might form the basis for developing hemorrhagic transformation and could cause excessive build-up of fluid leading to brain dysfunction or progression to chronic inflammation and formation of microthrombi in the subacute and chronic phases of stroke (Taheri et al., 2011; Yang and Rosenberg, 2011). Therefore, incomplete BBB recovery could represent a critical process for facilitating poststroke injury.

How BBB recovery occurs after stroke is still unclear. One hypothesized scenario suggests fast sealing of the barrier due to compensatory overexpression of TJ proteins, the presence of which can be found along blood vessels in stroke-injured tissue. However, in addition to protein expression, protein-protein interactions play a crucial role in TJ function. Therefore, BBB recovery is a complex process, the end effect of which is not just dependent on the expression pattern of key junctional proteins, but rather the degree of de novo organization of junction proteins. TJ disorganization produces increased paracellular permeability, the duration and magnitude of which may override any effect described as increased transcellular permeability.

The current study focused on defining changes in TJ complex organization and the mechanisms underlying potential TJ defects leading to prolonged BBB "leakage" affecting stroke recovery and long-term injury.

\section{Materials and Methods}

Brain tissue. Human postmortem brain tissue samples from stroke patients and healthy controls were from the Human Brain and Spinal Fluid Resource Center (West Los Angeles Healthcare Center, Los Angeles, CA) and the Mount Sinai National Institutes of Health (NIH) Brain Tissue Repository (New York, NY), which is sponsored by the National Institute of Neurological Disorders and Stroke/National Institute of Mental Health of the NIH, the National Multiple Sclerosis Society, and the Department of Veteran Affairs. Control brain slides were from ProSci Incorporated and had no stroke pathology. Details of the brain samples are included in Fig. 1-1 (available at https://doi.org/10.1523/ JNEUROSCI.1432-18.2018.f1-1). Ethical approval for these studies was obtained from the Institutional Review Board of the University of Michigan. Fresh frozen tissue was fixed with $4 \%$ paraformaldehyde and cryoprotected and cut at $15 \mu \mathrm{m}$; paraffin-embedded brain tissue was cut at 4 $\mu \mathrm{m}$. Stroke and control brain tissue were used for immunohistochemistry and PCR array analysis.

Transient middle cerebral artery occlusion ( $t M C A O)$. All experimental procedures were approved by the Institutional Animal Care and Use Committee of the University of Michigan. Experiments were performed on male 10- to 12-week-old C57BL/6 mice (The Jackson Laboratory). Mice were anesthetized with ketamine and xylazine $(100$ and $10 \mathrm{mg} / \mathrm{kg}$, i.p.). Body temperature was maintained at $37 \pm 0.5^{\circ} \mathrm{C}$ with a heating blanket and heating lamp during the entire experimental procedure. Focal cerebral ischemia was induced by right tMCAO using an intraluminal filament technique as described previously and confirmed by a laser Doppler flow probe. After $30 \mathrm{~min}$ of $\mathrm{tMCAO}$, mice were reperfused by suture withdrawal. Sham-operated animals underwent all procedures except the occlusion. Physiological parameters $\left(\mathrm{pO}_{2}, \mathrm{pCO}_{2}, \mathrm{pH}\right.$, blood glucose, cerebral blood flow) were monitored before, during, and after tMCAO. The reperfusion duration, 1-28 d, was chosen based on our evaluation of physiological parameters as well as the survival rate after tMCAO.

Neurological deficits were evaluated using a modified neurological severity score (mNSS) by blinded investigators. The mNSS rates neurological function on scale of 14 and includes motor (muscle status and abnormal movement), sensory (visual, tactile and proprioceptive), reflex, and balance tests. A corner test (sensorimotor functional test) was performed for each mouse at baseline and on days 1, 7, 14, 21, and 28 after tMCAO. The mice were allowed to walk into a corner $\left(60^{\circ}\right.$ angle $)$ and exit direction (right or left) was recorded by two independent investigators blinded to experimental design. Ten trials were performed (1 min between each) and percentage of ipsilateral (right) turns calculated.

Morphometric measurement of infarct volume. Animals were killed between 1 and $28 \mathrm{~d}$ after tMCAO and the brains removed, fixed, cryoprotected, and cryosectioned in the coronal plane. To measure infarct volume, sections from bregma were selected for Nissl and Fluoro-Jade B staining. Five coronal sections $(20 \mu \mathrm{m})$ at $2.0 \mathrm{~mm}$ intervals from bregma $(+4.0$ to $-6.0 \mathrm{~mm})$ were collected and stained with $0.1 \%$ cresyl violet (Sigma-Aldrich) according to standard protocol. For Fluoro-Jade (Millipore) staining, slides were incubated in Fluoro-Jade B diluted in $0.1 \%$ acetic acid for $30 \mathrm{~min}$ and rinsed and mounted.

Infarction area in each slice was determined by computerized image analysis system (Image J) and infarct volume calculated by multiplying by the distance between sections. Infarct volume was calculated as follows: [contralateral hemisphere volume - (ipsilateral hemisphere volume measured injury volume)]. All measurements were performed in a blinded manner and were analyzed separately by three independent researchers.

Permeability assay. In vivo BBB integrity was assessed by determining the transfer coefficient $\left(K_{\mathrm{i}}\right)$ for FITC-inulin $(5 \mathrm{kDa})$, FITC-dextran $(20$ 
$\mathrm{kDa})$, and/or Texas red-dextran (40 kDa) as described previously (Sladojevic et al., 2014). The tracer $K_{\mathrm{i}}$ was determined using an equation developed by Ohno et al. (1980).

The in vitro permeability of mBMEC monolayers was measured as described previously and modified in our laboratory (Kazakoff et al., 1995; Dimitrijevic et al., 2006). The permeability coefficient ( $\mathrm{cm} / \mathrm{min})$ of monolayers was calculated for the tracers FITC-inulin or Texas reddextran ( $1 \mu \mathrm{g} / \mathrm{ml}$; Sigma-Aldrich) at different reoxygenation time points for $0-48 \mathrm{~h}$.

Water content. Brain water content was measured by the wet/dry weight method. Ischemic and nonischemic hemisphere samples were weighed wet and then oven dried at $100^{\circ} \mathrm{C}$ for $48 \mathrm{~h}$ and reweighed. Brain water content (\%) was calculated as follows: [(wet weight - dry weight)/wet weight] $\times 100 \%$.

Mouse microvessel isolation. For analysis of ischemia/reperfusion (I/R) injury, brain microvessels from penumbra and ipsilateral hemisphere were isolated. Penumbra was marked as the area $1 \mathrm{~mm}$ in diameter around ischemic lesion collected by "pinch-out" method (Sladojevic et al., 2014). The corresponding contralateral region and contralateral hemisphere were also collected. For microvessel isolation brain tissue was mechanically dissociated and homogenized in a Dounce homogenizer. After washing with Hank's balanced solution, myelin and erythrocytes were cleaned with $18 \%$ dextran solution (Sladojevic et al., 2014). The isolated blood vessels were then digested for 5 min with $0.25 \%$ tryp$\sin$ at $37^{\circ} \mathrm{C}$ to remove perivascular cells. Microvessel purity was evaluated by immunocytochemistry using anti-CD31 (brain endothelial cells, 1:200; BD Bioscience), GFAP (astrocytes, 1:500; Sigma-Aldrich), PDGFR $\beta$ (pericytes, 1:100 Abcam), and Iba1 (microglia, 1:100; Abcam) antibodies. The protocol produced 99.99\% "clean" (without perivascular cells) blood vessels.

Mouse brain microvascular endothelial cell (mBMEC) culture. mBMECs were prepared using a modified protocol described previously (Sladojevic et al., 2014). Cells were selected with anti-CD31-coated magnet beads (Dynabeads). The protocol typically produces primary endothelial cell cultures that are $\sim 99 \%$ pure (determined by immunocytochemistry with an anti-CD31 antibody).

The mouse brain endothelial cell line (mBECL) was purchased from Angio-Proteomie and maintained as recommended by the manufacturer.

In vitro $I / R$ injury. $m B M E C$ and $m B E C L$ were subjected to oxygenglucose deprivation (OGD) injury (Dimitrijevic et al., 2006). Injury was initiated by placing cultures in an anaerobic chamber (Coy Laboratory) in the presence of OGD medium (DMEM glucose free solution purged with an anaerobic gas mixture, $5 \% \mathrm{CO}_{2} / 95 \% \mathrm{~N}_{2}$ and $10 \% \mathrm{H}_{2}$ removing residual oxygen) to induce an ischemia-like condition for $5 \mathrm{~h}$ at $37^{\circ} \mathrm{C}$. Then, cultures were removed from the chamber, medium exchanged with oxygenated DMEM, and cells placed in an incubator at $37^{\circ} \mathrm{C}$ with $5 \% \mathrm{CO}_{2}$-containing normal oxygen $\left(21 \% \mathrm{O}_{2}\right)$ to mimic reperfusion for 0-48 h. For every set of experiments, viability assays were performed (lactate dehydrogenase assay; Promega). Only cell cultures with cell viability of $>95 \%$ after OGD exposure were used in experiments.

Fusion proteins. Full-length open reading frame (ORF) claudin-1, claudin-5, and ZO- 1 were cloned out of commercially available untagged cDNA constructs. ORFs were cloned into pmCherry-C1 vector and pAcGFP-C1 infusion ready vector to create N-terminally fused fluorescent proteins (Takara). Plasmid DNA was isolated from individual transformed stellar competent (Takara) colonies using Qiagen plasmid midiprep kits. DNA sequences were validated with Sanger sequencing.

Cell treatment and transfection. Fusion proteins were single or double transfected in mBECs and mBECL using Torpedo ${ }^{\text {DNA }}$ (Ibidi). The expression profile/level of transfected proteins were evaluated after $5 \mathrm{~d}$ and/or after establishing a stable cell line. Claudin-1 depletion was achieved through transfection with either claudin-1 shRNA plasmid DNA $(0.5 \mu \mathrm{g}$ final concentration; Sigma-Aldrich) or claudin 1 siRNA (siclaudin1RNA \#si64036, \#64037, \#64038, each at $5 \mathrm{~nm}$ final concentration; Thermo Fisher Scientific) targeting three different claudin-1 regions for $24 \mathrm{~h}$. As controls, cells were transfected with nontarget shRNA control plasmid (0.5 $\mu \mathrm{g}$; Sigma-Aldrich) or with shRNA (sicontrol \#2, 5 nм; Thermo Fisher Scientific).
Claudin-1 peptide treatment. Claudin-1 peptide (C1C2) was synthesized as described previously (Staat et al., 2015). For in vitro experiments, claudin-1 peptide was dissolved in culture medium (DMEM) at $1 \mu \mathrm{g} / \mathrm{ml}$ and applied to mBMECs in two experimental conditions at 6 and $12 \mathrm{~h}$ of reperfusion. Cell viability assay indicated $>99 \%$ viability after treatment with claudin-1 peptide. In vivo, claudin-1 peptide was dissolved in sterile $0.9 \% \mathrm{NaCl}$ and injected intraperitoneally $(5 \mu \mathrm{g} / \mathrm{kg})$ at $3,5,7$, and $10 \mathrm{~d}$ after $\mathrm{tMCAO}$. Mice were randomly assigned to control peptide (cp) and claudin-1 peptide (C1C2) groups and treated with peptide $(5 \mu \mathrm{g} / \mathrm{kg})$ for 5 consecutive days during reperfusion starting 3 or $5 \mathrm{~d}$ after tMCAO. No side effects of claudin-1 and control peptide administration were observed.

Cell-fractionation, coimmunoprecipitation, and Western blotting. Cell fractionation and Western blot analysis was performed on isolated brain microvessels from infarcts surrounding area (penumbra) and mBMECs exposed to OGD reoxygenation conditions in vitro. Cell fractionation was performed using a ProteoExtract Subcellular Proteome Extraction kit (Calbiochem) to separate membrane, cytosolic, cytoskeletal, and nuclear fractions. Fraction specificity was confirmed using anti-cytochrome P450 reductase (membrane), anti-calpain (cytosole), and anti-vimentin (actin cytoskeletal fraction) antibodies. For "total cell lysate," cells were washed in PBS, scraped, and rinsed in lysis buffer.

Coimmunoprecipitation was performed using EZviewRed protein $\mathrm{G}$ affinity Gel beads (Sigma-Aldrich) with Western blotting performed with the following: anti-claudin-1, anti-claudin-5, ZO-1, and $\beta$-actin antibodies (all 1:1000 dilution; Cell Signaling Technology). Immunoblots were analyzed using ImageJ software.

Immunofluorescence and quantitative immunofluorescence. For immunofluorescence staining, brain section and cell samples were incubated with the following primary antibodies: claudin-5-Alexa Fluor 488conjugated (1:200 dilution), ZO-1-Alexa Fluor 594-conjugated (1:200), claudin-1-Alexa Fluor 488 (1:200; Life Science Technology) and antifibrinogen antibody (1:500; Abcam) and reactions visualized by Texas red-conjugated anti-sheep antibody. To measure fluorescent intensity in brain samples, three sections per stroke case and control slides were chosen and on each histological slide, 10 spots were randomly selected. Images (30 images per each staining/case) for quantitative florescence analysis were acquired on a confocal laser-scanning microscope (Nikon A1) with sequential mode to avoid interference between channels and saturation. Contrast, brightness, and the pinhole were held constant. Analysis was done in ImageJ software by outlining the fibrinogenpositive area and claudin-1- and claudin-5-positive staining in blood vessels in controls in each image and the fluorescence intensity in the enclosed area was measured. Background intensity was subtracted from the fluorescence intensity. Quantitation of TJ-associated fragments for claudin- 1 and claudin-5 were performed on 12 images obtained from three independent slides using ImageJ software.

Superresolution imaging. Superresolution imaging was done at Single Molecule Analysis in Real-Time (SMART) Center of the University of Michigan. mBMECs were exposed to OGD condition, transfected with claudin-1 and/or treated with peptide, and processed for immunofluorescence staining using the following primary and secondary antibodies: anti-claudin-1, claudin-5, and ZO-1-Alexa Fluor 647 (all 1:200 dilution; Invitrogen) and goat anti-mouse IgG Alexa Fluor Plus 647 (Thermo Fisher Scientific). Immediately before imaging, sample buffer was exchanged with STORM imaging buffer: $100 \mathrm{~mm}$ Tris-Cl, $25 \mu \mathrm{M} \mathrm{NaCl}, 1 \%$ v/v BME ( $\beta$-mercaptoethanol), $\mathrm{pH}$ 9.0, and freshly added $2.5 \mathrm{~mm}$ PCA (3,4-dihydroxybenzoic acid; Sigma-Aldrich, P5630) and $25 \mathrm{~nm}$ PCD (protocatechuate dioxygenase; Sigma-Aldrich, P8279). Images were collected in HILO (highly inclined laminated optical sheet microscopy) illumination with $641 \mathrm{~nm}$ laser excitation on an Olympus IX81 microscope with a cell^ TIRF module. Images were collected on an Andor iXon Ultra EMCCD camera. Images were processed using the ThunderSTORM plugin of ImageJ.

Real-time RT-PCR. Total RNA from human and mouse brain microvessels was prepared using TRIzol (Thermo Fisher Scientific). Singlestrand cDNA was synthesized using RT2 first strand kit (Qiagene). Analysis of junction protein mRNA expression in human and mouse 
stroke and control brain tissue was done using Cell Junction PCR array (Qiagen) and associated software.

To analyze the levels of claudin- 1 and claudin- 5 mRNA in human and mouse brain tissue isolated microvessels and mBMECs after I/R injury, TaqMan real-time PCR assay was performed using the Applied Biosystems 7500 Real Time PCR system. Single-strand cDNA was synthesized using the $\mathrm{RT}^{2}$ first strand kit. Real-time PCR was performed for claudin-1 and claudin-5 using commercially available primers (Qiagen). $\beta$-actin was used to normalize samples.

Fluorescence resonance energy transfer (FRET) and fluorescence recovery after photobleaching (FRAP) analysis. cis- and trans-interactions of claudin-1 and/or claudin-5, claudin-1/ZO-1, and claudin-5/ZO1 were assessed in BMECs transfected with combinations of fluorophore-tagged (AcGFP or mCherry) claudin-1, claudin-5, and/or ZO-1. Analyses were performed on a Leica SP5X Inverted 2-Photon FLIM Confocal microscope at the University Michigan Microscopy Imaging Laboratories using LAS-X software. Laser power and line intensities were as follows: FRET: white light laser at 70\%, red laser line at $587 \mathrm{~nm}, 40 \%$ intensity, green laser line at $488 \mathrm{~nm}, 30 \%$ intensity. Acceptor photobleaching was performed with laser lines 579, 587, and $595 \mathrm{~nm}$, each at $100 \%$ for $80 \mathrm{~s}$. FRET efficiencies were calculated as follows: [(donor ${ }^{\text {post }}-$ donor $\left.^{\text {pre }}\right) /$ donor $\left.^{\text {post }}\right]$. Claudin-1/claudin-1, claudin-5/claudin-5, and claudin-1/ claudin-5 cis-interactions were observed in cell cytosol and membranes using a CellMask Deep Red Plasma membrane stain. For claudin-1 and claudin-5, homophilic and heterophilic trans-interaction contact enrichment was quantified as fluorescence enrichment at cell-cell contacts.

FRAP analysis of claudin-1, claudin-5, and ZO-1 mobility was done with the following conditions: argon laser at 25\%, green laser line at 488 , $30 \%$ intensity, photobleaching performed with laser line 488 at $100 \%$. Laser power and laser line intensities were kept constant across all sample groups. FRAP values were calculated as follows: $\%$ recovery $=[(5 \mathrm{~min}$ postbleach fluorescence -0 min postbleach fluorescence)/prebleach fluorescence $]^{\star} 100$.

Controls included: FRET, efficiencies of control samples coexpressing empty AcGFP and mCherry vectors were collected and subtracted from experimental FRET efficiencies during analysis; FRAP, a background region of interest (ROI) was selected in non-GFP-positive region to assess nonspecific changes to fluorescence intensity and this background percentage recovery was subtracted from all ROIs analyzed.

Protein array. A mouse inflammatory antibody array kit (Ray Biotech) was used to simultaneously detect and semiquantify 42 cytokines/ chemokines in samples collected under in vitro conditions. Densitometric analysis was performed using ImageJ analysis software with protein array analyzer plugins.

Statistical analysis. Analyses were performed using GraphPad Prism 6.0 software. Unpaired, two-tailed Student's $t$ test with Welsh correction and one-way ANOVA with Tukey's, Sidak's, and Holman-Sidak post hoc tests were used to test group level differences. Chi-squared test was used for neurological scores. Correlations were analyzed with Pearson's correlation $\chi^{2}$ test. A probability value $<0.05$ was regarded as statistically significant.

\section{Results}

\section{BBB permeability in poststroke conditions}

The chronic phase after tMCAO in mice is characterized by gradual regression of the ischemic lesion, improved neurological function, and BBB recovery. The infarct was significantly reduced at day 7 compared with day 1 after tMCAO ( $p=0.0009,0.0007$, $0.00045,0.00041$, ANOVA, Holm-Sidak post test) followed by "stabilization" of lesion size from days 7-28 (Fig. 1A). Mice had a persistent neurological deficit and unilateral weakness (Fig. $1 B, C)$.

BBB permeability was examined 1-28 d after tMCAO by measuring the clearance of different sized tracers, inulin $5 \mathrm{kDa}$, and dextran 20 and $40 \mathrm{kDa}$. For the high-molecular-weight tracer, dextran $40 \mathrm{kDa}$, ipsilateral (ischemic) $\mathrm{BBB}$ hyperpermeability was only found at day 1 and 3 (Fig. 1-2A, available at https://
doi.org/10.1523/JNEUROSCI.1432-18.2018.f1-2). In contrast, such hyperpermeability persisted for inulin $5 \mathrm{kDa}$ and dextran 20 $\mathrm{kDa}$ into the chronic phase of stroke $(7,14,21$, and $28 \mathrm{~d}$; Fig. 1D). In particular, the ipsilateral hemisphere showed a steady and significant increase in inulin permeability after tMCAO compared with ipsilateral hemisphere in sham controls and the contralateral hemisphere $(p=0.000038 ; 0.000032,0.000056$, and 0.000081 , ANOVA Holm-Sidak post test). There was only significant ipsilateral brain edema at day 1 and 3 post-MCAO, with resolution in late after stroke conditions (day 7-28; Fig. 1-2 B, available at https://doi.org/10.1523/JNEUROSCI.1432-18.2018.f1-2).

A parallel analysis of BBB integrity under chronic poststroke conditions in human brain showed similar changes. BBB leakage in poststroke conditions was evaluated in 5 brain samples from patients with previous stroke onset ( 6 months to 2 years) and 4 samples from patients without a history of stroke corresponding in age and gender (Fig. 1-1, available at https://doi.org/10.1523/ JNEUROSCI.1432-18.2018.f1-1). The stroke injury areas had robustFig. 1E). Semiquantitative analysis showed high level of extravascular fibrinogen in stroke patients pinpointing BBB leakage in chronic poststroke conditions $(t=5.713, p=0.0004$ unpaired $t$ test with Welch correction; Fig. $1 F$ ).

\section{$\mathrm{BBB}$ TJ protein changes in poststroke conditions}

To define potential BBB structural alterations during chronic poststroke conditions, we performed an expression microarray analysis of TJ proteins in murine and human brains. Isolated microvessels from the ipsilateral ischemic hemisphere after tM$\mathrm{CAO}$ and sham controls and brains from patients with chronic stroke and corresponding controls, were pooled to perform analysis (Fig. 1-1, available at https://doi.org/10.1523/JNEUROSCI. 1432-18.2018.f1-1). A total of 84 TJ-related genes were analyzed (i.e., cell surface adhesion, receptors, junctional interaction proteins, cytoskeleton regulators, and signaling proteins). In human samples, 52 genes were altered (upregulated or downregulated) in chronic poststroke conditions. In mouse, 50 genes were altered $21 \mathrm{~d}$ after tMCAO. In mice, the number of altered genes did not vary significantly with duration after tMCAO (54 at $7 \mathrm{~d} ; 52$ at $14 \mathrm{~d} ; 50$ at $28 \mathrm{~d}$; data not shown). Of the total genes altered in mouse and human, 34 were common for both mouse and human. Sixteen and 18 were specific for human and mouse, respectively (Fig. 2A). Scatter plots revealed that in ipsilateral hemisphere of mice $21 \mathrm{~d}$ after stroke, 45 genes were upregulated and only 5 genes were downregulated significantly (fold change $\geq 2$ ) compared with controls. In contrast, in human brain, there was 35 genes upregulated and 17 genes downregulated (Fig. 2B). Selected structural TJ genes (including transmembrane, scaffolding, and cytoskeleton proteins) are summarized in Figure $2 \mathrm{C}$. Interestingly, the ischemic hemisphere in mice and human showed upregulation of claudin-1, claudin-3, and claudin-12, as well as two transmembrane TJ proteins involved in the leukocyte recruitment, JAM-A and ESAM (Fig. 2-1A, available at https:// doi.org/10.1523/JNEUROSCI.1432-18.2018.f2-1). Claudin-5, a major occlusion protein, and occludin showed $>2$-fold downregulation in human and mouse brain (Fig. $2 C$ and Fig. 2-1 A, available at https://doi.org/10.1523/JNEUROSCI.1432-18.2018. f2-1).

\section{Claudin-1 and claudin-5 alterations in poststroke conditions in vivo}

Claudin-5 is a major protein in the BBB TJ, where it nearly completely occludes the paracellular space. Other claudins described in brain endothelial cells are claudin-3, present mostly 
A
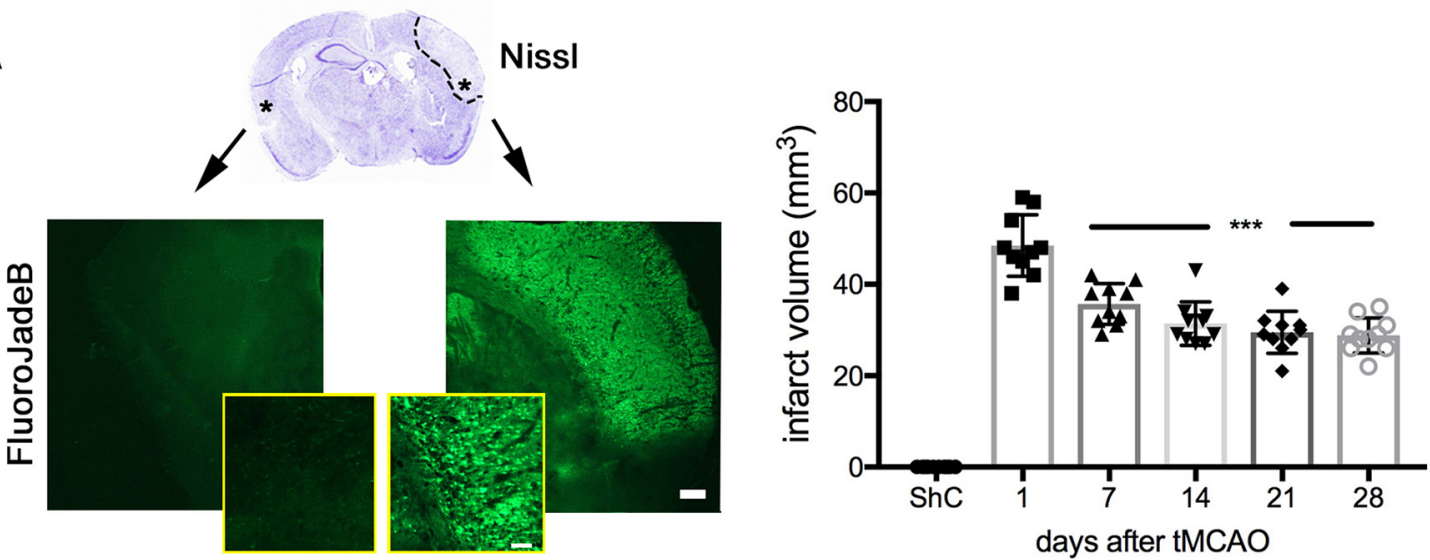

B
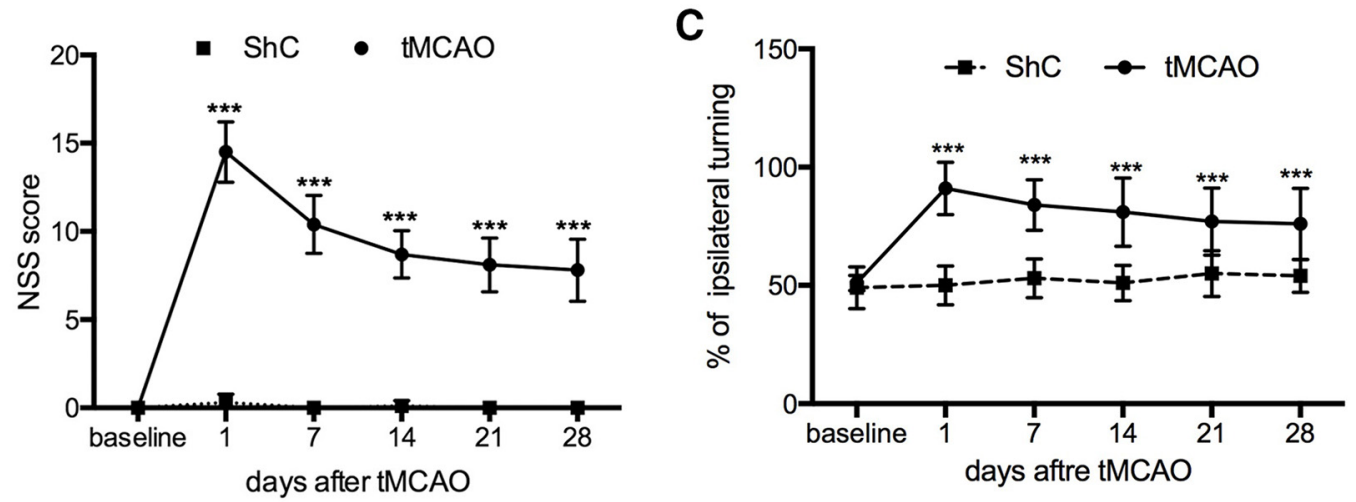

D
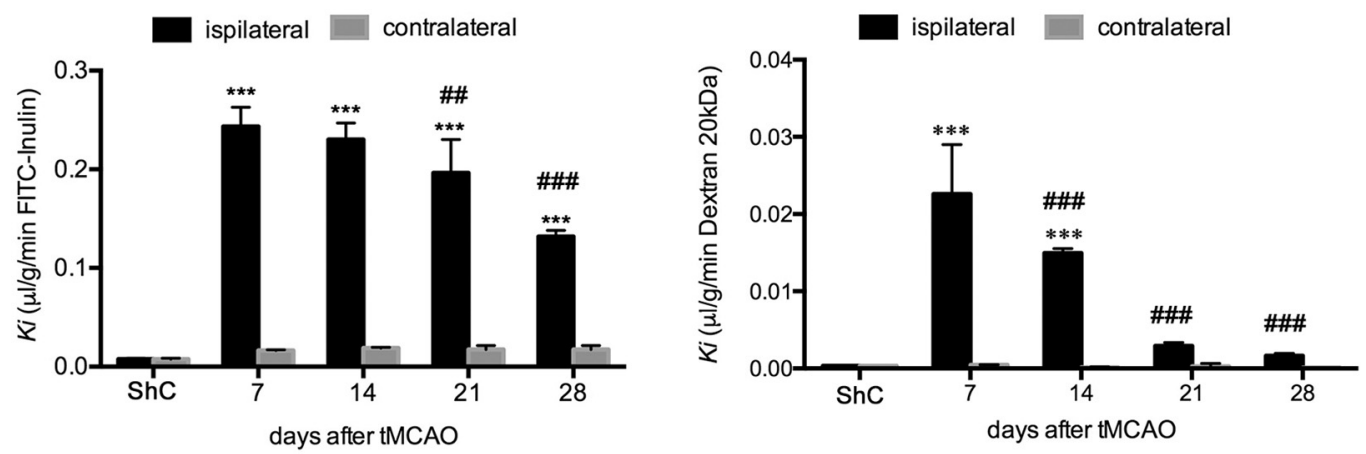

$\mathbf{E}$
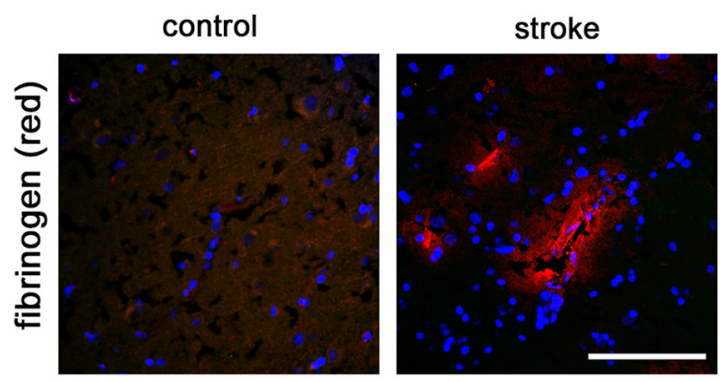

$\mathbf{F}$

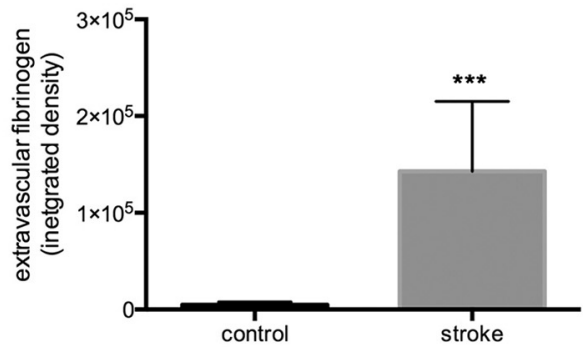

Figure 1. BBB leakage in chronic poststroke conditions in mice and patients. A, Representative images of Nissl and Fluoro-Jade B staining in infarct area at day 14 after tMCA0. Asterisk indicates corresponding area of ischemic and contralateral side in consequent slides stained with Fluoro-Jade B. Scale bar, $100 \mu \mathrm{m}$. The bar graph shows the brain infarct size assessed by morphometric analysis of Fluoro-Jade staining on histological sections at days 1, 7, 14, 21, 28 after tMCA0. Data are shown as mean \pm SD. $n=10$ each group. ${ }^{* * *} p=0.0009,0.0007,0.00045$, and 0.00041 , ANOVA, Holm-Sidak post test, compared with day 1. ShC, Sham control. $\boldsymbol{B}, \boldsymbol{C}$, Analysis of NSS (B) and corner turn test $(\boldsymbol{C})$ at days $1,7,14,21$, and 28 after tMCA0 in mice. Note the persistent neurological deficit in mice with tMCAO compared with ShC. Data are shown as mean \pm SD. $n=10$ each group; NSS ( $p=0.999{ }^{* * * *} p=0.0001,0.00045,0.00045,0.00054,0.0005$, ANOVA Sidak's post test); corner test $\left(p=0.9954, p=0.00067,0.00062 .0 .00073,0.00073,0.00075\right.$, ANOVA, Sidak's post test). $\boldsymbol{D}, K_{\mathrm{i}}$ for inulin $5 \mathrm{kDa}$, dextran $20 \mathrm{kDa}$, and dextran $40 \mathrm{kDa}$ (Figure $1-2$, available at https://doi.org/10.1523/JNEUROSCl.1432-18.2018.f1-2) in ischemic and nonischemic hemispheres at days 7-28 after transient MCA0. Data are shown as mean $\pm . n=8$; ${ }^{* * *} p=0.000003$, $0.000003,0.000006,0.00001$ for inulin and $p=0.000007,0.00008$ for dextran (ANOVA, Sidak's post test) comparing with ipsilateral hemisphere of Shc; \#\# $p=0.00264$, and ${ }^{\# \# \# ~} p=00009$ for inulin and $p=000094,0.000007,0.000007$ for dextran (ANOVA, Sidak's post test) compared with $K_{\mathrm{i}}$ value of ipsilateral hemisphere on day 7 after tMCA0. E, Examples of immunofluorescence staining for fibrinogen (red) in brain for from a control and a chronic stroke patient (clinical patients data are included in Figure 1-1, available at https://doi.org/10.1523/JNEUROSCI.1432-18.2018.f1-1). Blue DAPI staining indicates nuclei. Note the increased permeability of the blood vessels (leak to fibrinogen) in the stroke lesion. $\boldsymbol{F}$, Bar graph represents semiquantitation of the (Figure legend continues.) 

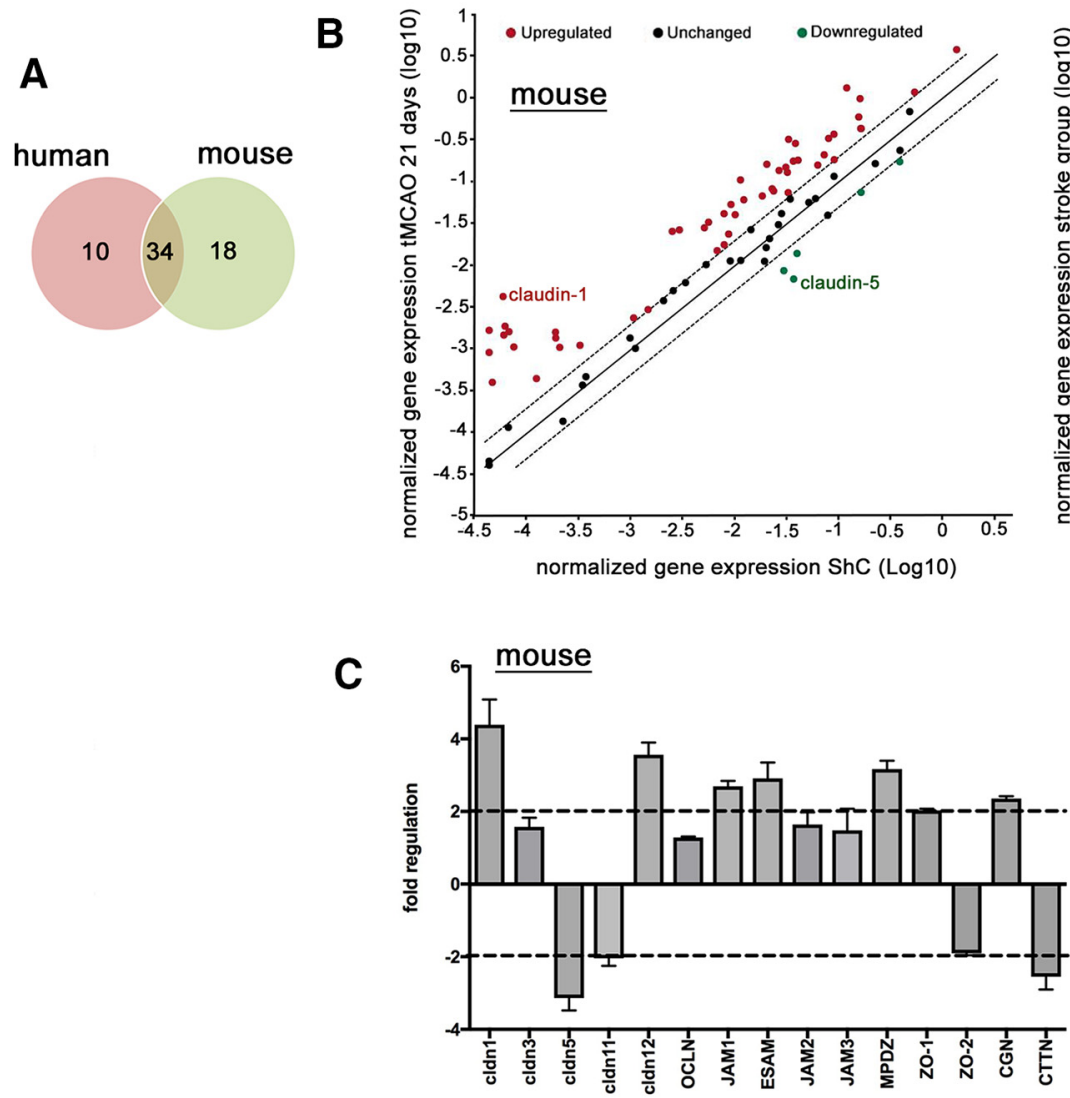
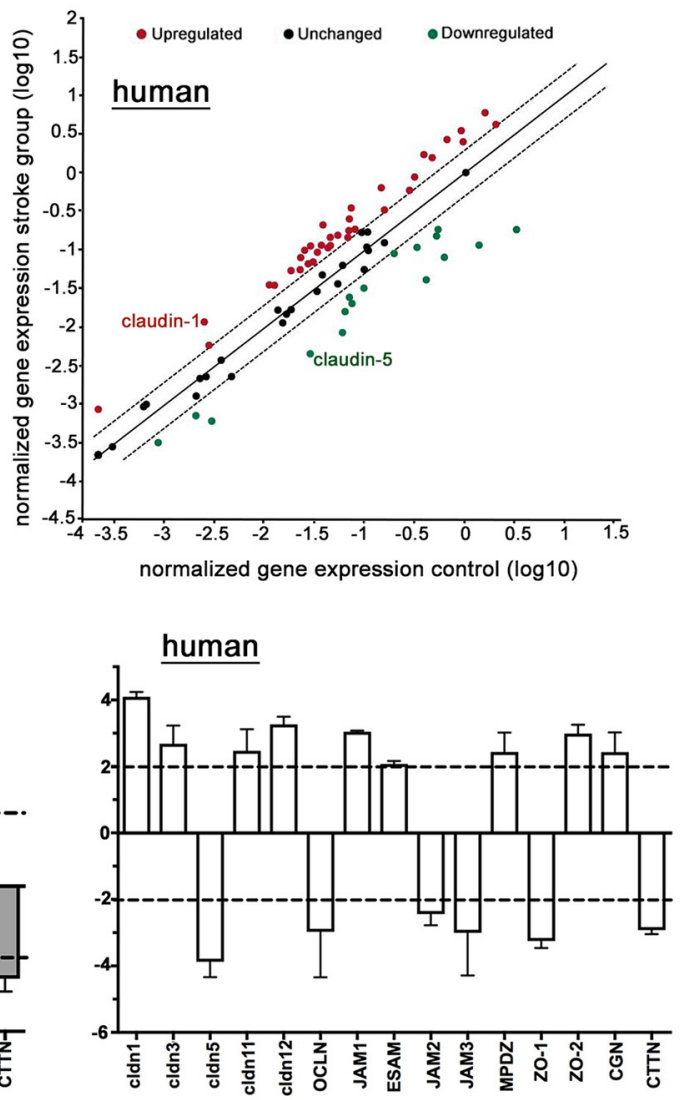

Figure 2. Alterations in the gene expression for TJ-associated proteins in brain microvessels under chronic poststroke conditions. $\boldsymbol{A}$, Venn diagram of total number of TJ-associated proteins in microvessels isolated from ischemic hemispheres where gene expression was altered at least twofold after human stroke and mouse tMCA0 (21 d). $\boldsymbol{B}$, Scatter plots showing the relationship between the mRNA expression of TJ associated proteins in control and ischemic microvessels. Expression was normalized to panel of housekeeping genes included in plate. Left, Mouse microvessels isolated from ischemic hemispheres tMCA0 (21 d) compared with sham controls (ShC). Right, Human microvessels from stroke patients compared with nonstroke (control) patients. Changes in expression with ischemia were classified as upregulated or downregulated if there was a $>2$-fold change. C, Bar graph of fold changes for representative structural TJ proteins in human and mouse microvessels isolated form ischemic hemispheres after human stroke and mouse tMCAO (21 d). Real-time RT-PCR analysis of claudin-1 and claudin-5 mRNA in blood vessels isolated from human brain patients with chronic stroke condition or control patients conformed the array results (Figure 2-1, available at https://doi.org/10.1523/JNEUROSCI.1432-18.2018.f2-1). The dashed lines represent the cutoff for upregulated and downregulated genes. OCLN, Occludin; ESAM, endothelial cell-selective adhesion molecule; MPDZ, multiple PDZ domain protein; CGN, cingulin; CTTN, cortactin.

developmentally, claudin-12, of unknown function, and in human brain endothelial cell lines, claudin-1 (Ohtsuki et al., 2007). The expression pattern of claudin- 1 and claudin-5 mRNA and protein in blood vessels was analyzed in chronic poststroke conditions in both mouse and human. In mouse ipsilateral hemisphere $7,14,21$, and $28 \mathrm{~d}$ after tMCAO, brain microvessels showed increased expression of claudin-1 mRNA ( $p=0.000003$, 0.000002, 0.0000038, and 0.00008; ANOVA Holman-Sidak's post test, compared with ipsilateral hemisphere of sham control; Fig. $3 A)$ and protein $(p=0.000001,0.0000009,0.00003$, and 0.00007; ANOVA Holman-Sidak post test; comparing with ipsilateral hemisphere of sham control; Fig. $3 B$ ). The contralateral hemisphere of mice with ischemic injury ( $\mathrm{tMCAO}$ ) did not show any increased in expression of claudin- 1 on mRNA and protein level evaluated at 7-28 d after injury. Among other cells in the neurovascular unit, astrocytes have been reported to express claudin-1. However, no claudin-1 staining was found in astro-

$\leftarrow$

(Figure legend continued.) extravascular accumulation of serum protein fibrinogen in brain. Data are shown as mean \pm SD of stroke cases $(n=150 ; 10$ analyzed blood vessels on 3 separate slides in 5 stroke cases) and control cases ( $n=120 ; 0$ analyzed blood vessels on 3 separate slides in 4 control cases). ${ }^{* *} p=0.0004 t=5.713$, unpaired $t$ test with Welch correction) stroke versus control brain. cytes in the current study (Fig. 3-1, available at https://doi.org/ 10.1523/JNEUROSCI.1432-18.2018.f3-1). Claudin-1 protein expression was closely associated with persistent vascular leakage to inulin (Pearson correlation $r=0.921$; Fig. 3C). Brain microvessels also had decreased expression of claudin-5 mRNA and protein in ipsilateral hemisphere at days 7, 14, 21, and 28 after MCAO (Fig. $3 A, B$ ). Altered claudin-5 protein expression was negatively associated with increased leakage to inulin (Pearson correlation $r=-0.906$; Fig. $3 C$ ). These results pinpoint that increased BBB leakage in the postischemic hemisphere might be correlated with an imbalance in claudin expression, increased claudin-1, and decreased claudin-5.

A similar pattern was also found in human brain in chronic poststroke conditions. Blood vessels from stroke but not control patients expressed claudin-1 mRNA and had reduced expression of claudin-5 (Fig. 2-1A, available at https://doi.org/10.1523/ JNEUROSCI.1432-18.2018.f2-1). At the protein level, vessels that were leaky to fibrinogen after stroke had increased claudin-1 expression and decreased claudin-5 expression compared with control brain (Fig. 3D). There was a significant positive correlation between claudin-1 protein expression and fibrinogen leakage (Pearson coefficient $r=0.9123$ ) and a negative correlation between claudin-5 expression and that leakage (Pearson coefficient $r=-0.7363$; Fig. $3 D)$. Therefore, as in mouse, there is an 
A
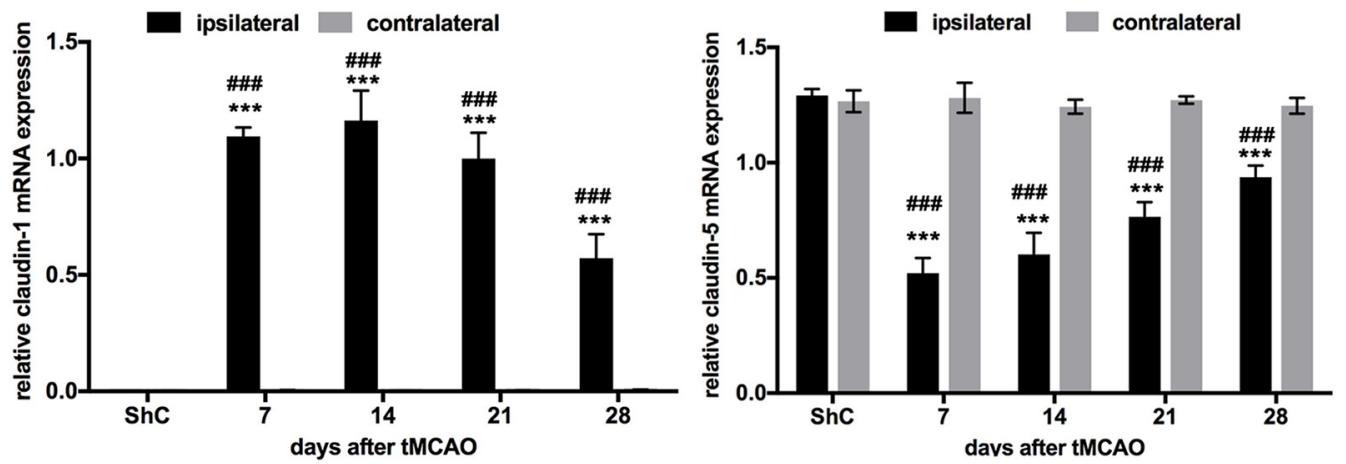

B

claudin-1

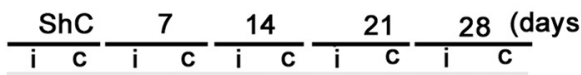

claudin- 5

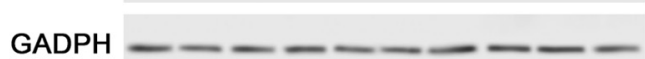

$$
\text { ipislateral contralateral }
$$

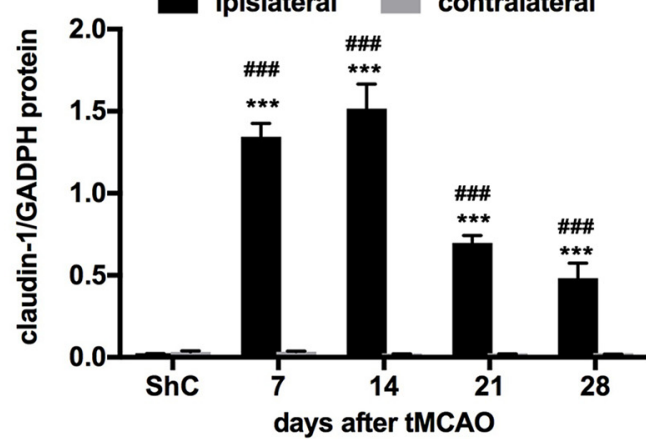

C

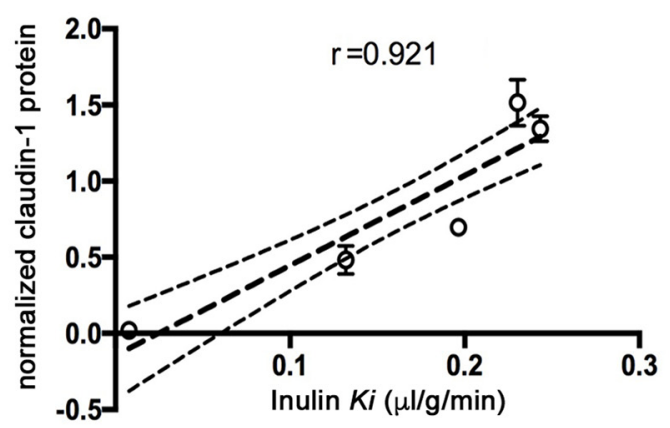

D

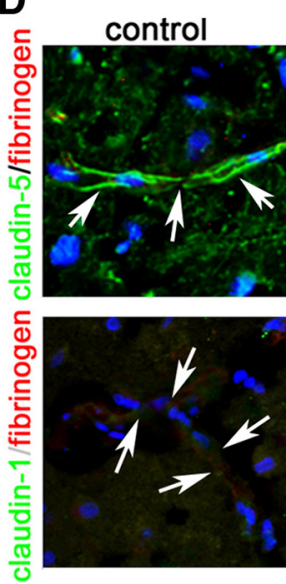

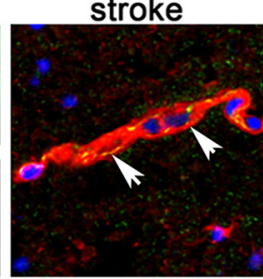
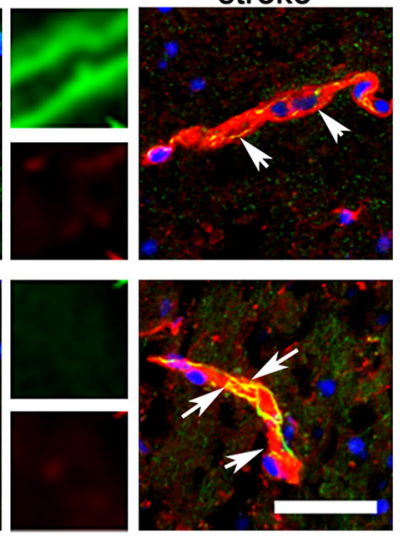

GADPH -------
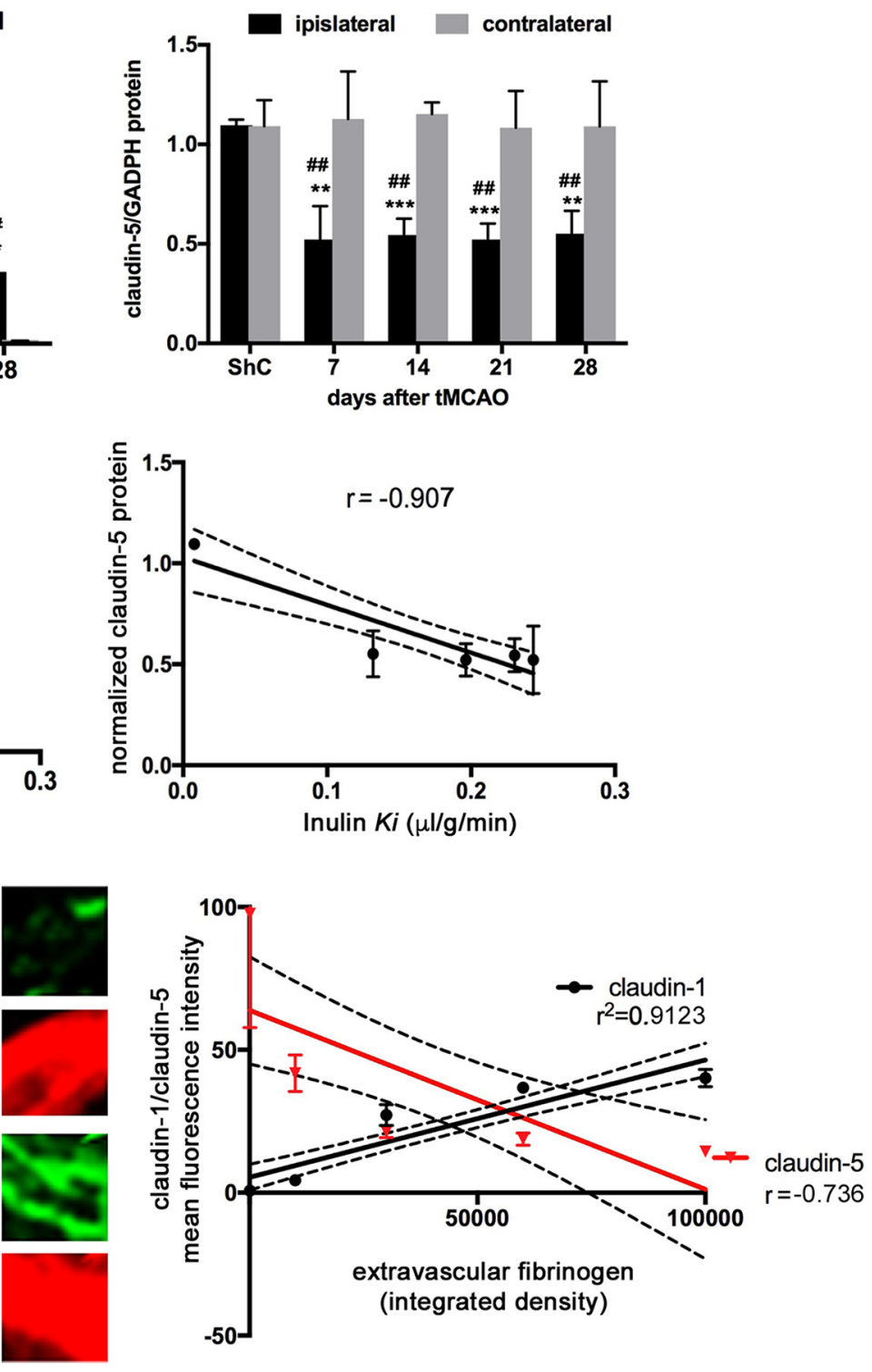

Figure 3. Claudin-1 is expressed in leaky blood vessels in chronic poststroke condition. A, Real-time RT-PCR analysis of claudin-1 and claudin-5 mRNA expression in isolated penumbral blood vessels after tMCAO compared with contralateral hemisphere and sham controls (ShC). mRNA levels were normalized to GAPDH. Notice that there was steady elevation of claudin-1 expression in ipsilateral hemisphere after stroke. In contrast, claudin-5 was downregulated compared with ShC. Data are shown as mean \pm SD. $n=5 . p=0.999,{ }^{* * *} p=0.000001,0.0000008,0.000001$, 0.000008 -claudin-1; $p=0.987, p=0.0006,0.00005,0.00007,0.000089$ - claudin-5 (ANOVA, Sidak's post test) comparing with ipsilateral and contralateral hemisphere and ${ }^{\# \# \#} p<0.0000001$, $0.0000002,0.0000006,0.000006,0.00002,0.000023,0.000071,0.000089$ (both claudin-1 and claudin-5, ANOVA, Sidak's post test) comparing with ipsilateral hemisphere (Figure legend continues.) 
imbalance in claudin- 1 and claudin- 5 in human brain in chronic poststroke conditions.

\section{Effect of claudin-1 on barrier properties after oxygen glucose deprivation in vitro}

Similar to in vivo, there was increased expression claudin-1 mRNA and protein 24 and $48 \mathrm{~h}$ after OGD in vitro (Fig. 2-1 B, available at https://doi.org/10.1523/JNEUROSCI.1432-18.2018. f2-1). I/R injury in vitro is associated with persistent permeability of brain endothelial cell monolayers to dextran $20 \mathrm{kDa}$ and inulin $5 \mathrm{kDa}$ with very limited alterations in cell density and viability $(<1 \%)$. Depleting claudin-1 by shRNA before I/R injury facilitated a faster recovery of barrier properties protecting barrier integrity in the chronic phase ( 24 and $48 \mathrm{~h}, p=0.000001$ and $p=$ 0.000043 , ANOVA Holm-Sidak post test; Fig. 4A). In addition, overexpressing claudin- 1 alone caused brain endothelial barrier hyperpermeability (unpaired $t$ test, $t=12.77, p=0.00021$; Fig. $4 B)$.

\section{Claudin-1 distribution in brain endothelial cells and integration into the $\mathrm{TJ}$ complex}

In brain endothelial cells after I/R injury in vitro and tMCAO in vivo showed $\sim 45 \%$ of claudin- 1 was in the membrane fraction, $50 \%$ in the cytosol, and a small portion in the nuclear fraction (only 5\%; Fig. 4C). A similar distribution was found in claudin1-overexpressing cells (Fig. 4C). Morphologically, membraneassociated claudin-1 was localized at cell-cell contacts (Fig. $4 D, E)$. The dynamics of claudin- 1 incorporation into the TJ complex were further analyzed by FRAP. Claudin-1-mCherry fusion protein had relatively low mobility in postischemic conditions, similar to claudin-5-AcGFP, suggesting incorporation into cell junctions (Fig. 4F; for specificity of fusion proteins, see Fig. 5-1A, available at https://doi.org/10.1523/JNEUROSCI.143218.2018.f5-1).

The incorporation of transmembrane proteins into TJs depends on establishing interactions with scaffolding proteins such as ZO-1 and ZO-2. Immunofluorescence (Fig. 5A) and immunoprecipitation analysis (Fig. $5 C$ ) showed claudin-1 colocalized

\section{$\leftarrow$}

(Figure legend continued.) in ShC. B, Western blot and semiquantitative densitometric analysis of claudin-1 and claudin-5 protein expression in isolated penumbral blood vessels after TMCAO and corresponding area in the nonischemic contralateral hemisphere and sham controls. Protein levels were normalized to GAPDH. Notice the decreased expression of claudin-5, whereas claudin-1 showed increased expression after tMCA0. Data are shown as mean $\pm . n=$ 5; claudin-1, $p=0.9998,{ }^{* * *} p=0.000001,0.000002,0.000001,0.00003$, and 0.00004; claudin-5, $p=0.999,{ }^{* *} p=0.003,0.00039,0.0003$, and 0.0012 comparing ipsilateral and contralateral hemisphere (ANOVA Holm-Sidak post test); claudin-1, ${ }^{\# \#} p=0.000001$, $0.000002,0.000008$, and 0.00001 and claudin-5, ${ }^{\# \# p}=0.0031,0.003,0.0037$, and 0.0032 comparing with ipsilateral hemisphere in ShC (ANOVA Holm-Sidak post test). Western blotting image is one of five independent experiments. $\boldsymbol{C}$, Correlation between claudin- 1 or claudin-5 expression and BBB permeability (inulin $K_{\mathrm{i}}$ ) at the different time points. Pearson coefficient for claudin-1, $r=0.921, p=0.0262$; for claudin-5, $r=-0.907 ; p=0.0336$. D, Immunofluorescence staining of claudin-5 or claudin-1 (green) and fibrinogen (red) in a human brain from a control patient and one with chronic stroke. Arrow indicates one analyzed microvessel split in two channels (zoom images) to demonstrate the decreased claudin-5 and increased claudin-1 expression in fibrinogen leaky vessels and absence of claudin-1 expression in the cells in neurovascular units (astrocytes) (Figure 3-1, available at https://doi.org/10.1523/JNEUROSCI.143218.2018.f3-1). Quantification of the claudin-1 and claudin-5 fluorescent signals was performed in ImageJ. Comparison of claudin-1 and claudin-5 expression (mean fluorescent intensity) and blood vessel leakiness (integrated density of extravascular fibrinogen). A Pearson correlation coefficient showed high correlation between claudin-1 expression and vessel leakiness $(r=0.9123, p=$ $0.0308)$ and a negative correlation between claudin- 5 expression and leakiness $(r=-0.7362$, $p=0.159)$ in chronic stroke brain tissue. Scale bar, $50 \mu \mathrm{m}$. predominantly with ZO-1 but not ZO-2 at the border of brain endothelial cells after $\mathrm{I} / \mathrm{R}$ injury and during claudin-1 overexpression. FRET analysis of fusion proteins claudin-1-AcGFP and ZO-1-mCherry revealed that claudin-1 interacted with ZO-1 with a FRET efficiency of $0.4 \pm 0.1$, comparable to claudin-5/ ZO-1 interaction (FRET efficiency $0.7 \pm 0.1$. Fig. $5 B$; for specificity of fusion proteins, see Fig. 5-1 A, available at https://doi.org/ 10.1523/JNEUROSCI.1432-18.2018.f5-1, and Fig. 5-1 B, available at https://doi.org/10.1523/JNEUROSCI.1432-18.2018.f5-1). A ZO-2/ claudin-1 interaction was not present (data not shown).

\section{Homophilic claudin-1 interactions and heterophilic interactions with claudin-5}

Cis- and trans-claudin interactions at the plasma membrane are critical for making a stable and functional TJ complex. Claudin-1 has the ability to establish predominantly homophilic transinteractions in brain endothelial cells at a similar magnitude to claudin-5/claudin-5 trans-interactions (Fig. 5E). Claudin-1/ claudin-5 trans-interactions were less prevalent (Fig. 5E), although immunocytochemistry indicated points of colocalization between claudin-1 and claudin-5 (Fig. 5D). Similarly, cisclaudin-1 interactions were predominantly homophilic (claudin1/claudin-1) at the cell membrane and cytosol as determined by FRET efficiency (Fig. 5F). Although there was some heterophilic cis-interaction with claudin-5, this was significantly less than homophilic claudin-1 and homophilic claudin-5 cis-interactions $(p=0.005$ for membrane and $p=0.000001$ for cytosol, ANOVA Holm-Sidak post test; Fig. $5 F$ ). This suggests that claudin-1 can incorporate into brain endothelial TJs by establishing stable interaction with ZO- 1 and building claudin- 1 strand by establish claudin-1/claudin-1 cis- and trans-interactions.

\section{Effects of claudin- 1 on claudin-5/claudin-5 and claudin-5/ ZO-1 interactions}

Claudin-1 might increase brain endothelial barrier permeability by altering the interactions of other proteins within the TJ complex. We examined the effect of claudin-1 on the homophilic trans-interaction of claudin-5 and the interaction of claudin-5 with ZO-1. Claudin- 1 affected the mobility of claudin- 5 evaluated by FRAP analysis in brain endothelial cells exposed to $\mathrm{I} / \mathrm{R}$ injury as well as during claudin-1 overexpression (claudin $1^{\text {over }}$ ). Depletion of claudin-1 via shRNA rescued claudin-5 incorporation in the TJ complex (Fig. 6A). The claudin-1 effect on claudin-5 distribution was further confirmed by cell fractionation. Claudin- 5 was redistributed from the cell membrane and accumulated in cell cytosol when claudin-1 was increased after I/R injury, tMCAO, or directly overexpressed (claudin- ${ }^{\text {over }}$ mBECL) (Fig. 6B).

Furthermore, cells overexpressing claudin- 1 had fragmented claudin-5 staining at endothelial cell-cell borders (Fig. 6C). Evaluating the length of claudin- 5 fragments in the two experimental conditions, we found significant reduction in claudin-5 length $(p<0.000001$, ANOVA, Holm-Sidak post test), but not complete loss (Fig. 6D). Complete depletion of claudin-1 by shRNA rescued claudin- 5 expression and cell border localization in $\mathrm{I} / \mathrm{R}$ condition (Fig. 6B,C). (Fig. 6-1 $A-B$, available at https://doi.org/ 10.1523/JNEUROSCI.1432-18.2018.f6-1). In addition, claudin-5 trans-interaction, measured by FRET analysis of a mixed culture of claudin-5AcGFP and claudin-5-mCherry cells, was significantly diminished in the presence of claudin- 1 ( $p=0.000002$, ANOVA Holm-Sidak post test), indicating that claudin-5 can still form claudin-5 trans-interactions, but this was interrupted with claudin-1/claudin-1 trans-interactions, generating probably 
A

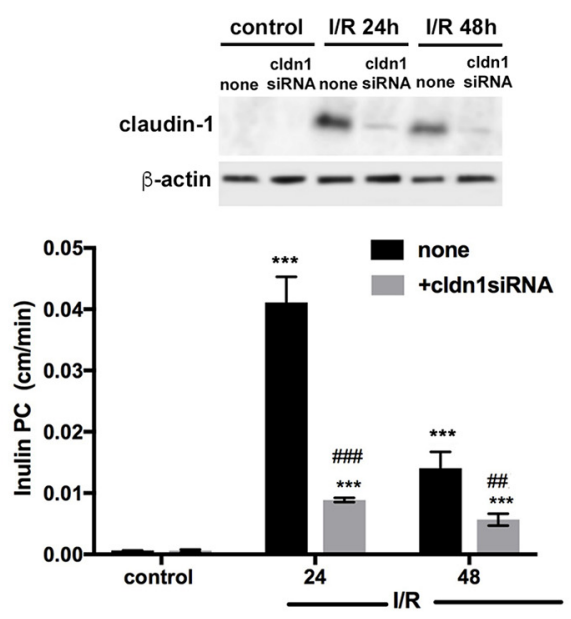

C

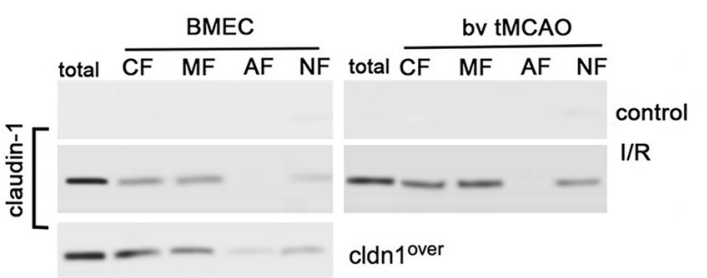

B
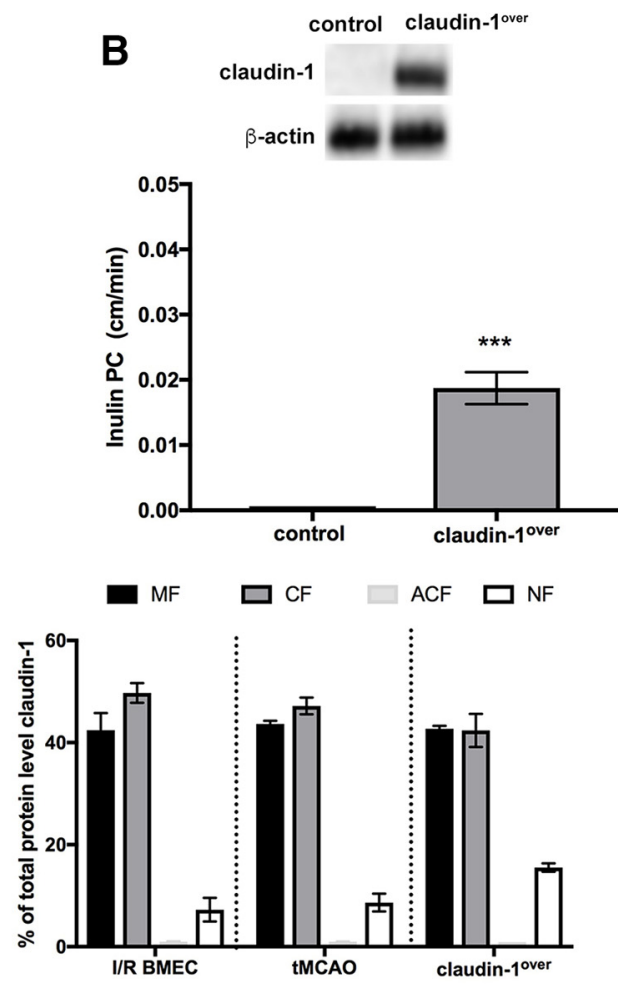
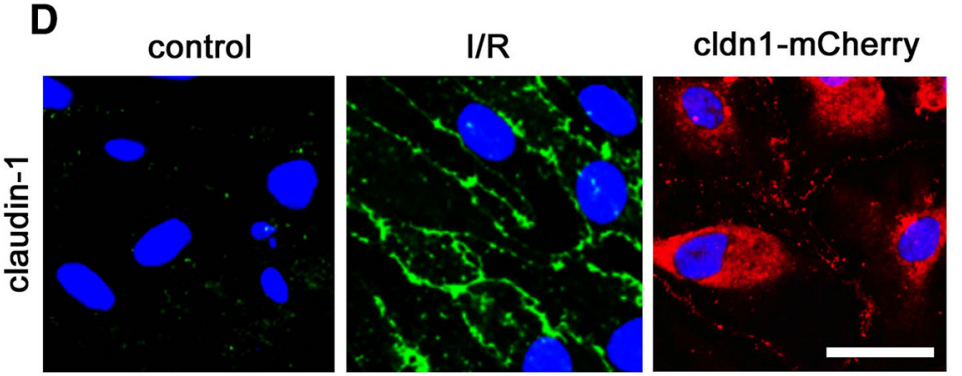

$\mathbf{F}$

$\mathbf{E}$
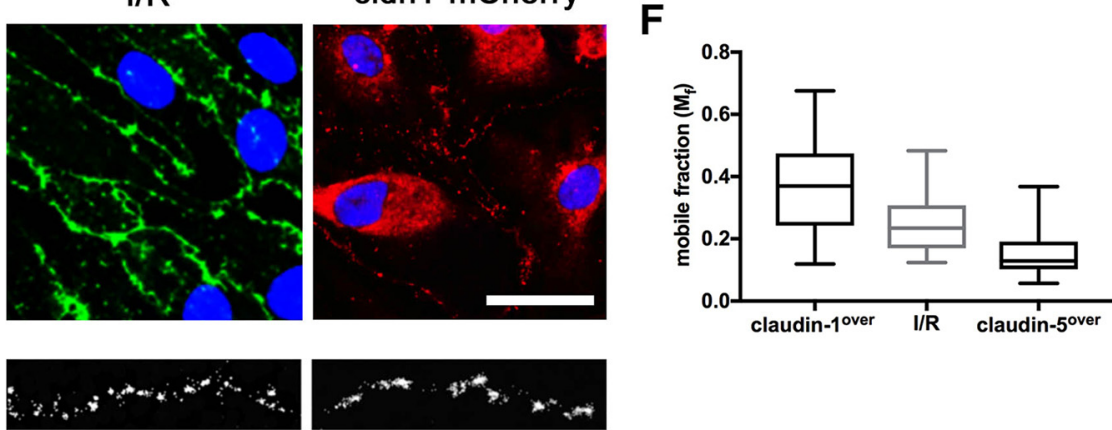

Figure 4. Claudin-1 alters brain endothelial barrier permeability. $A$, Permeability coefficient (PC) for FITC-inulin in mBMEC monolayers exposed to I/R injury for 24 and $48 \mathrm{~h}$. Depletion of claudin-1 by siRNA (cldn1siRNA) had a protective effect reducing the brain endothelial barrier hyperpermeability after I/R. Data are shown as means $\pm S D . n=5 ; p=0.9997,{ }^{* * *} p=0.0000001,0.000001$, and 0.000043 compared with control and ${ }^{\# \# \#} p=0.0000001,{ }^{\# \#} p=0.00102$ comparing the barrier permeability between cells with/without depletion of claudin 1 by siRNA (ANOVA, Sidak's post test) Western blotting analysis showed knock-down efficiency of claudin-1 siRNA in experimental groups. Representative image of Western blotting from five independent experiments is shown. $B$, Claudin-1 overexpression in BECL increased the permeability to FITC-inulin. Western blot data are shown as means \pm SD. $n=8 ; t=12.77,{ }^{* * *} p<0.0002$ compared with control (unpaired $t$ test). Representative image of Western blotting from eight independent experiments showed efficiency of claudin-1 overexpression in BECL. C, Cell fractionation and Western blot analysis of the cellular distribution of claudin-1 in BMECs and isolated penumbral blood vessels during reperfusion and in BECLs overexpressing claudin-1 (cldn 1 over). At 24h after 0GD in BMECs, $7 \mathrm{~d}$ after tMCA0 in blood vessels (bv) and in cldn ${ }^{\text {over }}$ BECL, claudin-1 was primarily present in the membrane fraction (MF) and cytosolic fraction (CF). Little was present in the actin cytoskeletal fraction (ACF) or nuclear fraction (NF). "Total" represents the total protein fraction of claudin-1 analyzed in the cell fractionation assay. Representative images of Western blot of three independent experiments are shown. The bar graph shows the percentage of claudin-1 in the different cell fractions. Data are shown as means \pm SD. $n=3$. D. Claudin-1 expression and localization in BMECs analyzed by immunofluorescence staining in control and I/R conditions ( $24 \mathrm{~h}$ reoxygenation). There was increased claudin- 1 after I/R injury and it was present at cell- cell borders. Overexpression of claudin- 1 in BECL by transfection with cldn 1-mChery showed a similar pattern of claudin-1 distribution at the cell border as well as some cytosolic expression. Scale bar, $20 \mu \mathrm{m}$. E, Superresolution images of TJ-associated claudin-1 fragments on the cell border in control, I/R injury and BMEC-claudin-1 ${ }^{\text {over }}$ cells. F, FRAP analysis to assess the claudin-1 (Cldn1) mobile fraction in mBMECs overexpressing claudin-1 (claudin-1 ${ }^{\text {over}}$ ) and mBMECs exposed to I/R injury (24 $\mathrm{h}$ reoxygenation). The results indicated low mobility (e.g., potential incorporation into the TJ complex). BMEC-claudin-5 $5^{\text {over }}$ represents cells transfected with AcGFP-cldn5 and is included as a positive control. Data are shown as means \pm SD. $n=30$ ROls per group.

mixed homophilic strands of claudin-1 and claudin-5 in brain endothelial cells (Fig. 6E). Moreover, claudin-1 significantly reduced claudin-5/ZO- 1 interaction $(p=0.0021$ and $p=0.0027$, ANOVA Holm-Sidak post test) and this was rescued with claudin-1 depletion (Fig. 6F).

\section{Targeting claudin-1 to improve BBB recovery after stroke}

The effect of claudin-1 on brain endothelial monolayer leakage after I/R injury suggested that it might be a good target for reduc- ing I/R-induced brain endothelial hyperpermeability. In vitro, targeting claudin- 1 by adding claudin-1 C1C2 peptide $24 \mathrm{~h}$ after OGD exposure reduced monolayer permeability (Fig. $7 A, B$ ) that was time and dose dependent (Fig. 7-1 A, available at https:// doi.org/10.1523/JNEUROSCI.1432-18.2018.f7-1 1B; for specificity of C1C2 peptide, see Fig. 7-1C, available at https://doi.org/ 10.1523/JNEUROSCI.1432-18.2018.f7-1). C1C2 peptide also had morphological effects establishing continuous claudin-5 strands in both claudin-1-overexpressing cells and mBMECs ex- 
A
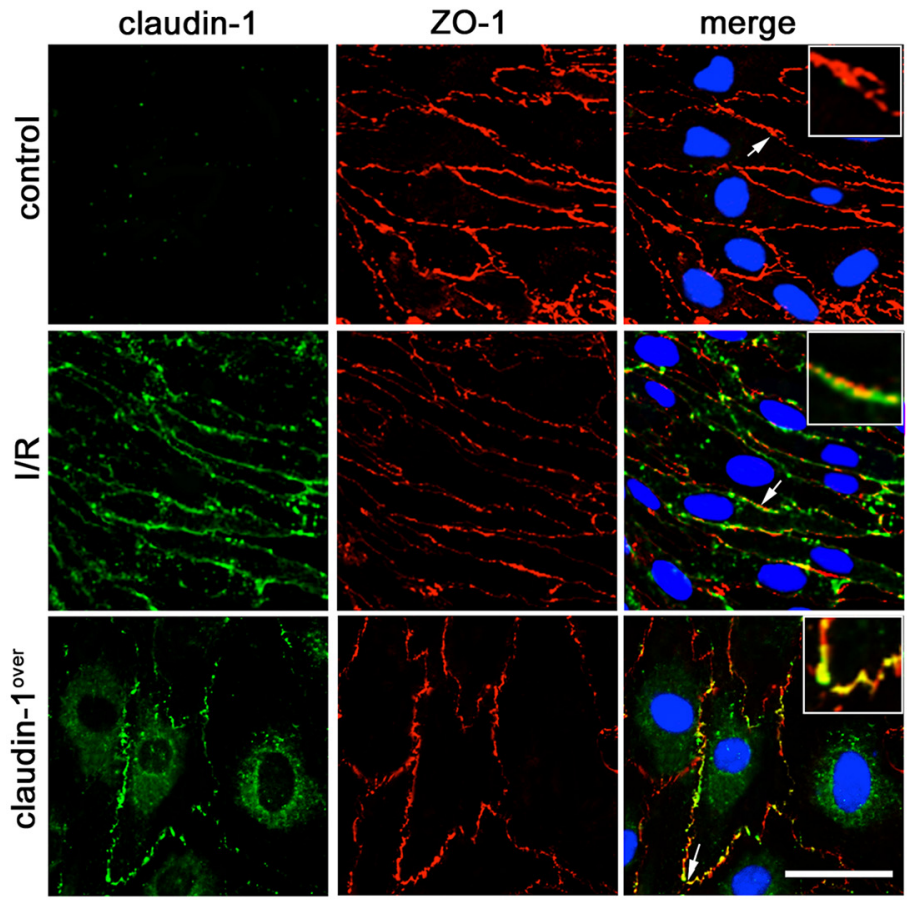

D
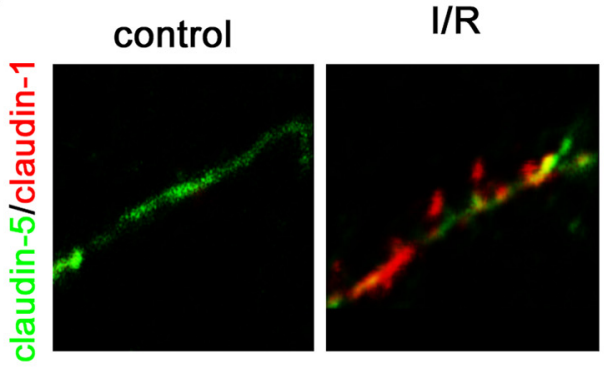

F
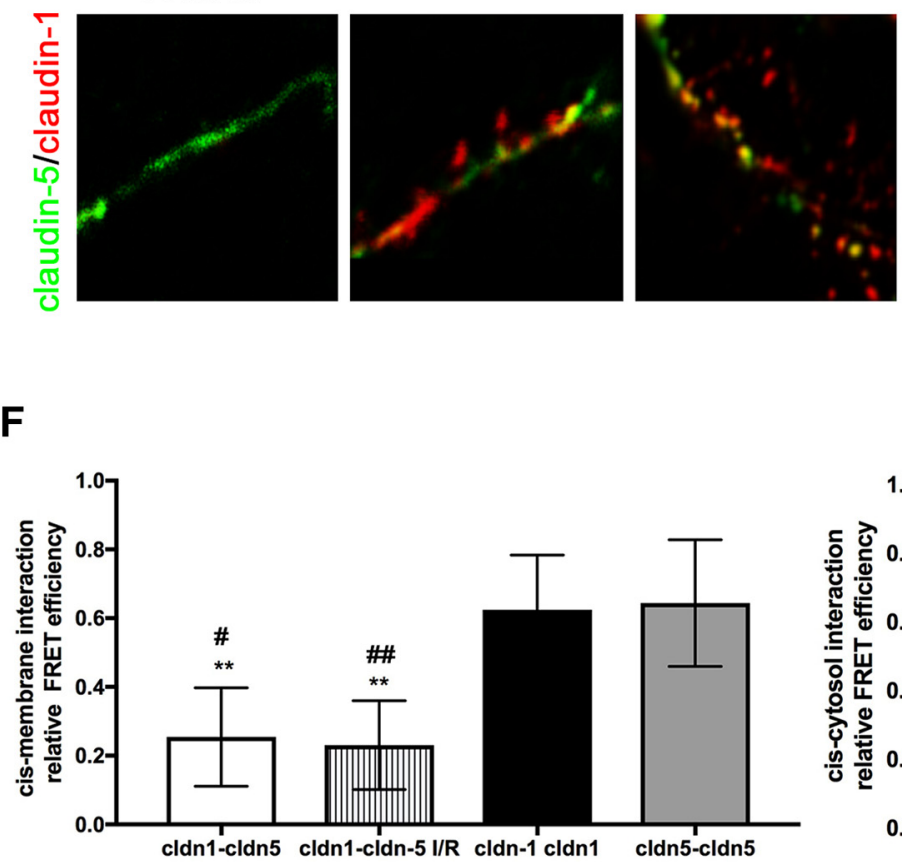

B
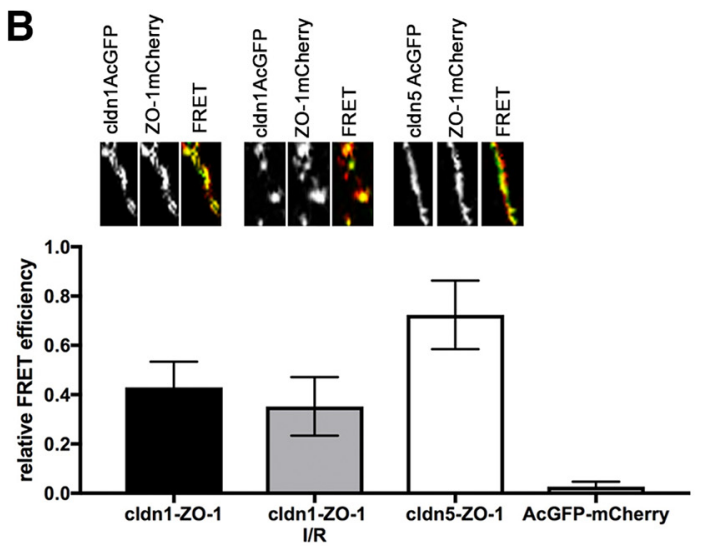

C

$\frac{\text { control }}{\text { input }} \frac{\mathrm{I} / \mathrm{R}}{\text { input }} \frac{\text { claudin-1 }}{\text { input }}$

claudin-1

ZO-1

E

IP:ZO-1
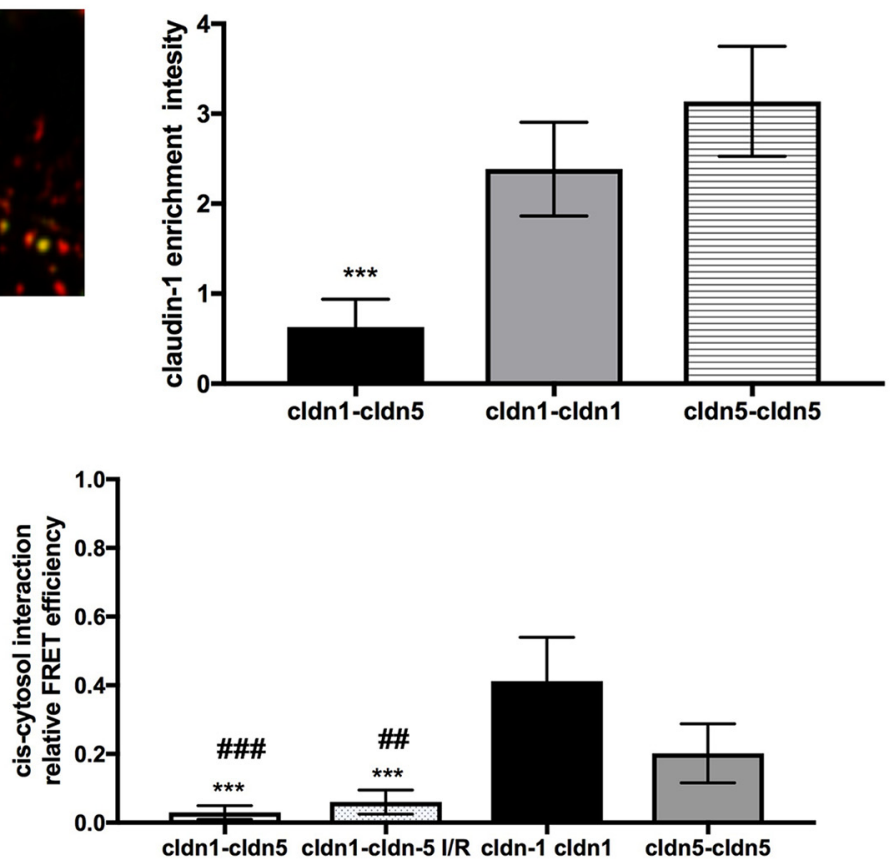

Figure 5. Claudin-1 incorporation into the TJ complex. A, Immunofluorescence staining for claudin-1 and Z0-1 in control, I/R-exposed cells (48 h reoxygenation), and claudin-1-overexpressing cells (Claudin-1 ${ }^{\text {over }}$. Arrow and magnified images (box) indicate colocalization of ZO- 1 and claudin- 1 on the cell border. $B$, Acceptor photobleaching FRET analysis of claudin- 1 associated ZO-1 in cells coexpressing claudin-1AcGFP and Z01-mCherry (fusion proteins specificity in Figure 5-1A, available at https://doi.org/10.1523/JNEUROSCl.1432-18.2018.f5-1, and Figure 5-1B, available at https://doi.org/10.1523/JNEUROSCI.1432-18.2018.f5-1) in control and I/R conditions. Positive and negative controls represent cells cotransfected with CIdn5-AcGFP and Z0-1 mCherry and AcGFP and $\mathrm{mCherry} \mathrm{vectors.} \mathrm{Data} \mathrm{are} \mathrm{shown} \mathrm{as} \mathrm{means} \pm$ SD. cells. $n=30$ ROIs/group. C, Immunoprecipitation (IP) and Western blot analysis of claudin-1 and Z0-1 interaction/colocalization in control, I/R exposed cells and claudin-1-overexpressing cells (Claudin- $1^{\text {over }}$ ). Representative images of Western blot of three independent experiments are shown. $\boldsymbol{D}$, Example of immunofluorescence staining of claudin-1 and claudin-5 at cell-cell borders in control, I/R-exposed cells, and cells overexpressing claudin-1 (Claudin-1 ${ }^{\text {over }) . ~ E, ~ A c c e p t o r ~ p h o t o b l e a c h i n g ~ F R E T ~ a n a l y s i s ~ o f ~ c l a u d i n-1 / ~}$ claudin-1, claudin-1/claudin-5, and claudin-1/claudin-5 trans-interactions indicated predominantly homophilic claudin- 1 and claudin-5 interactions (rather than claudin-1/claudin-5). Data are shown as means \pm SD. cells $n=30$ ROls/group, ${ }^{* * *} p=0.000023$ comparing homophilic claudin-1 and claudin- 5 with heterophilic claudin-1/claudin 5 trans-interaction (ANOVA, Sidak's post test). $F$, Acceptor photobleaching FRET analysis of cis-claudin- 1 and claudin- 5 interaction at the cell membrane and cytosol in control and I/R condition (24 h). Data are shown as means \pm SD. cells. $n=$ 20 Rols/group. ${ }^{* *} p=0.0051$, and 0.0051 for cis-membrane and ${ }^{\# \# \#} p=0.000001,0.000001$ for cis-cytosol claudin 1 - claudin5 interaction compared with homophilic claudin-1; $p=0.0156$ for cdn1-cldn5 I/R compared with cldn5-cldn5, \# $p=0.0051,0.004$ for cis-membrane and ${ }^{\# \#} p=0.00073,0.0063$, for cis-cytosol cldn1-cldn5 interaction comparing with homophilic claudin-5 cis-interaction (ANOVA, Holm-Sidak post test). 
A

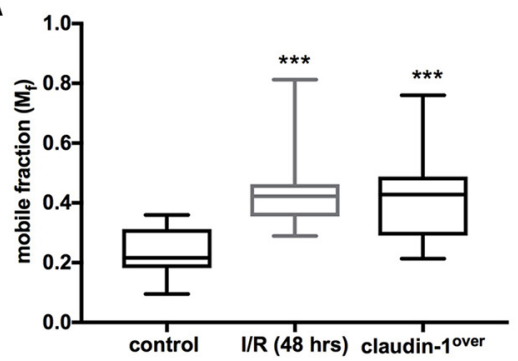

B

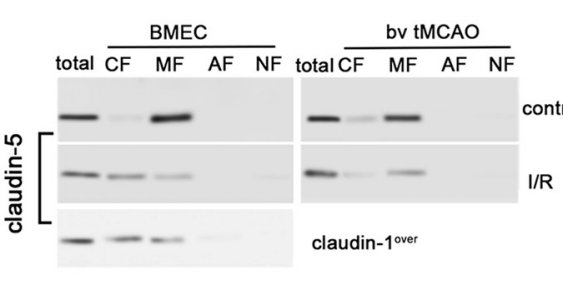

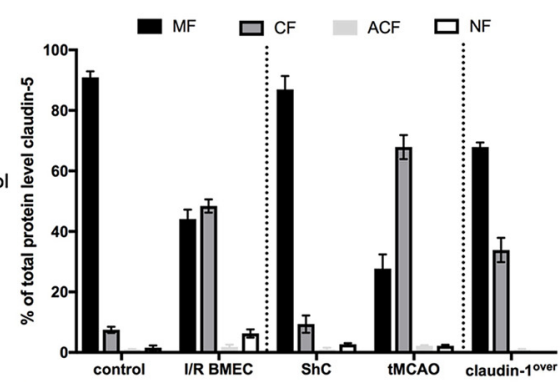

C control
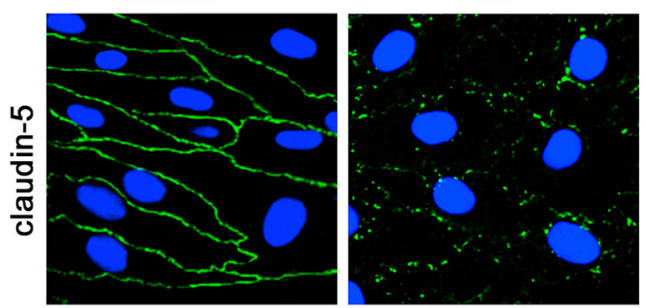

I/R+claudin1shRNA
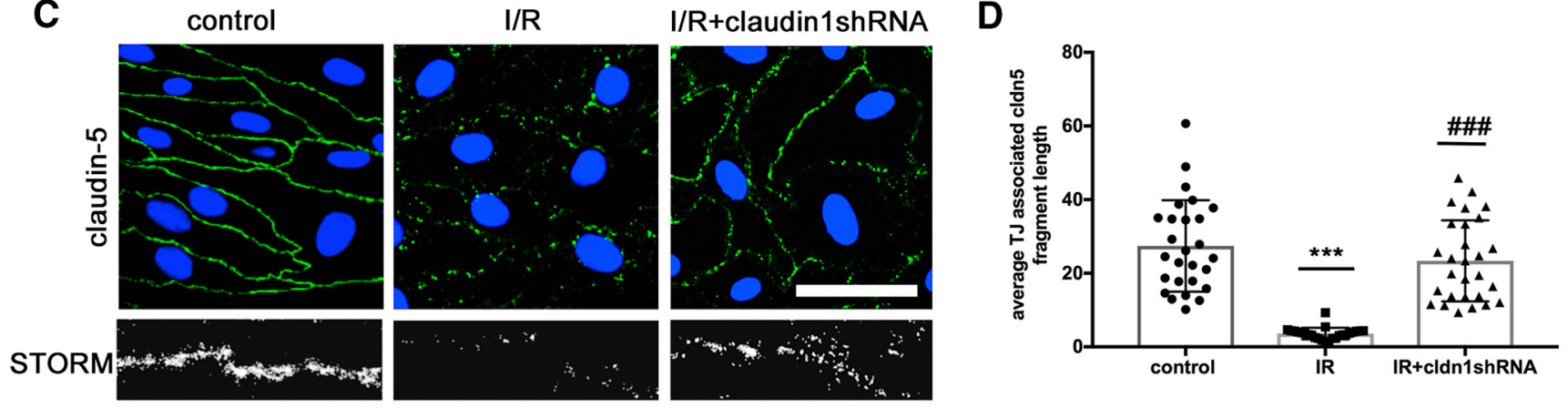

E

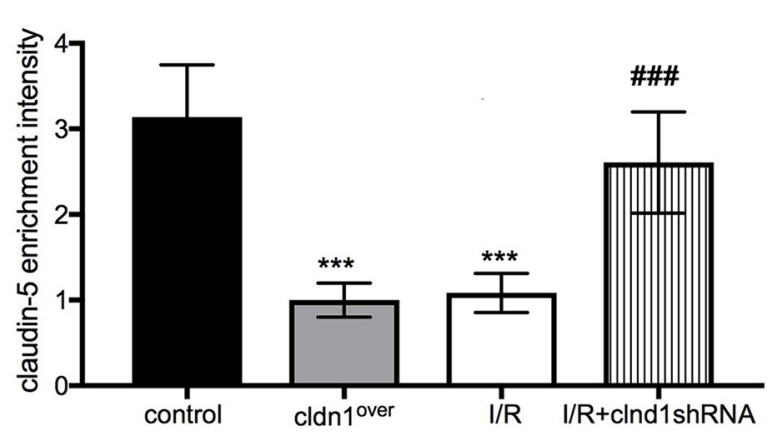

$\mathbf{F}$

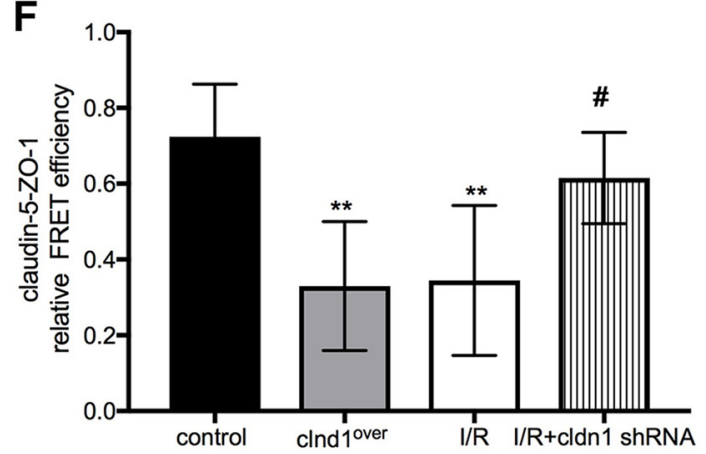

Figure 6. Claudin-1 effects on claudin-5 TJ incorporation. A, FRAP analysis of TJ-associated claudin-5 in control, I/R exposed cells (48 h) and claudin-1 overexpressing BMEC (claudin- ${ }^{\text {over }}$ ) ${ }^{* *} p=0.00023,0.000035$ compared with control (ANOVA, Tukey's post test). B. Cell fractionation and Western blot analysis of claudin- 5 in mBMECs and isolated penumbral blood vessels during reperfusion showed claudin-5 distribution of in membrane, cytosol, and nuclear fraction at time points $48 \mathrm{~h}$ (mBMEC) and $7 \mathrm{~d}$ (blood vessels) as well as claudin- 1 overexpressing BMEC. MF, CF. and ACF indicate membrane, cytosolic, and actin cytoskeletal cell fractions, respectively. "Total" represents the total protein fraction of claudin-5 analyzed in the cell fractionation assay. Representative images of Western blot of three independent experiments are shown. Bar graph shows the percentage of claudin-5 distributed through the fractions. Data are shown as means \pm SD. C, Immunofluorescence staining and superresolution images of TJ-associated claudin-5 in control and I/R exposed BMECs with and without depletion of claudin-1 by claudin-1 shRNA. Scale bar, 20 $\mu \mathrm{m} . \boldsymbol{D}$, Quantitation of the average TJ-associated claudin-5 fragment length in claudin-5/Z0-1 costained immunofluorescent images in control and I/R-exposed cells with and without depletion of claudin-1 by claudin-1 shRNA (for knock-down efficiency see Figure 6-1, available at https://doi.org/10.1523/JNEUROSCI.1432-18.2018.f6-1). ${ }^{* * *} p=0.000001$ and $p=0.2737$ compared with control; $\# \#=0.00001$ compared with I/R-exposed cells (ANOVA, Tukey's post test). $E$, FRET analysis (enrichment intensity) of the claudin-5 trans-interaction in control, cldn $1{ }^{\text {over }}$ BMECS, and I/R-exposed cells with and without depletion of claudin-1 with cldn1 shRNA. Data are shown as means \pm SD. cells. $n=30$ ROls per group. ${ }^{* * *} p=0.000001$ and $p=0.000001$ compared with control and ${ }^{\# \# \#} p=0.000001$ and to I/R (ANOVA Tukey's test). $F$, FRET analysis of claudin-5 and Z0-1 interaction in control, cldn 1 overBMEC, and I/R-exposed cells with and without depletion of claudin-1 with cldn1shRNA. ${ }^{* *} p=0.00219,0.002708,0.6476$ compared with control; $\# p=0.0299$ compared with I/R (ANOVA, Tukey's test).

posed to I/R injury as evaluated by STORM imaging (Fig. 7C). The $\mathrm{C} 1 \mathrm{C} 2$ peptide also reduced claudin-1/ZO-1 interaction and facilitated claudin-5/ZO-1 interaction (Fig. 7C). Importantly, in vivo, administering claudin-1 C1C2 peptide daily starting from day 5 after tMCAO decreased BBB leakage to inulin $(5 \mathrm{kDa})$ from day 7 ( $\sim 2$-fold, $p=0.00008)$ and progression to day $28(\sim 3.6-$ fold, $p=0.000001$; Fig. $7 F)$. Therefore, improved BBB permeability was associated with improved neurological function in chronic stroke (Fig. 7G).

\section{Claudin-1 alters brain endothelial cell}

inflammatory phenotype

We also investigated whether claudin-1 has additional brain endothelial barrier effects in chronic I/R injury conditions. Because
I/R injury induces proinflammatory mediator expression, we screened alterations in the brain endothelial inflammatory phenotype after I/R injury. Claudin-1 overexpression caused increased ( $>2.5$-fold) expression of CXCL13, CCL11 CX3CL1, GM-CSF, IL-1 $\beta$, IL6, and CCL5 in cell culture medium (Fig. $7 E$ ). The expression of these cytokines/chemokines by brain endothelial cells during I/R injury was reduced if claudin-1 expression was depleted by shRNA or if claudin-1 C1C2 peptide was added during reperfusion (Fig. 7E). Therefore, claudin-1 may regulate brain endothelial cell phenotype as well as impairing barrier integrity by affecting barrier structural organization. Claudin-1 depletion may have beneficial effects on poststroke BBB permeability and consequently on chronic poststroke injury. 
A

C

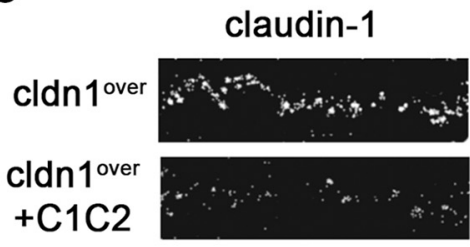

D
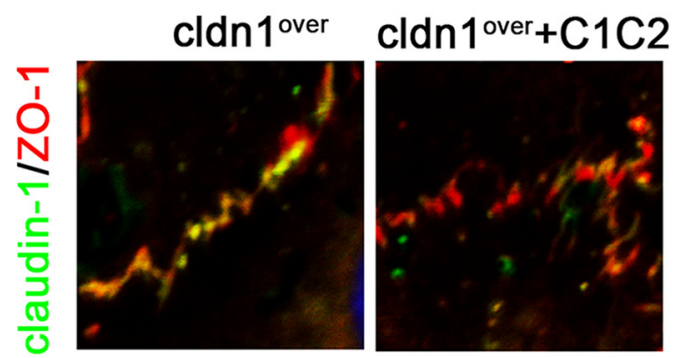

claudin-5

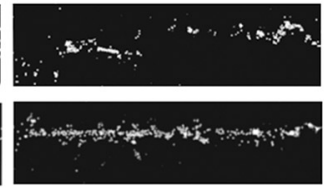

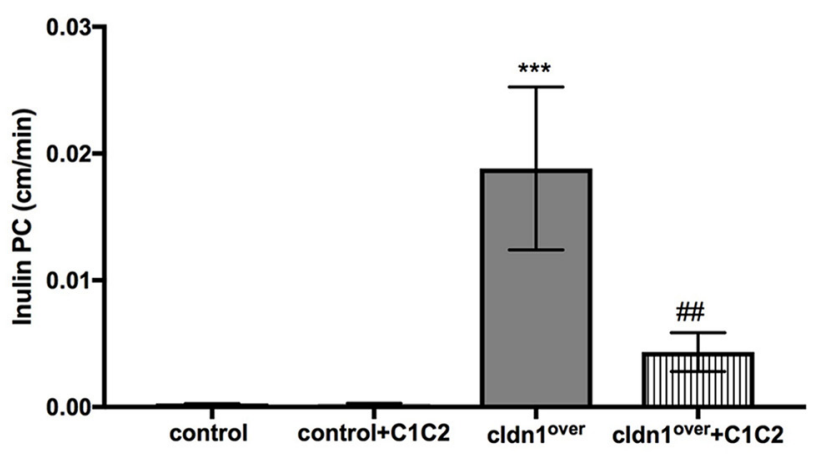

B

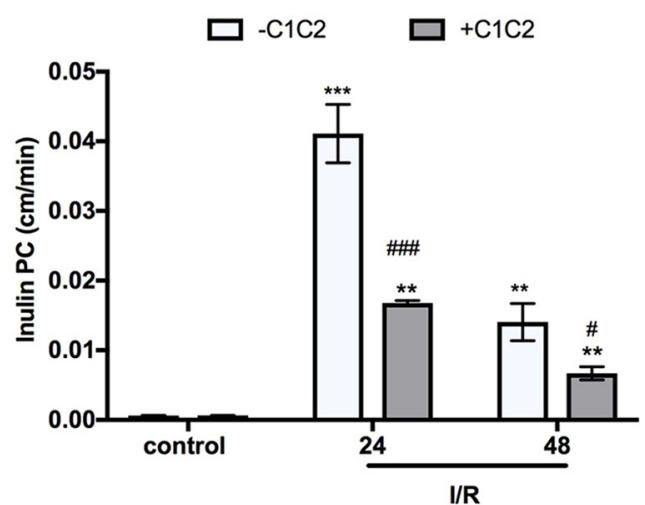

control $\square$ cldn10ver $\square$ IR

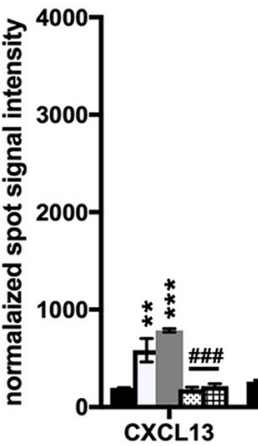

$\square$ cld

E

F

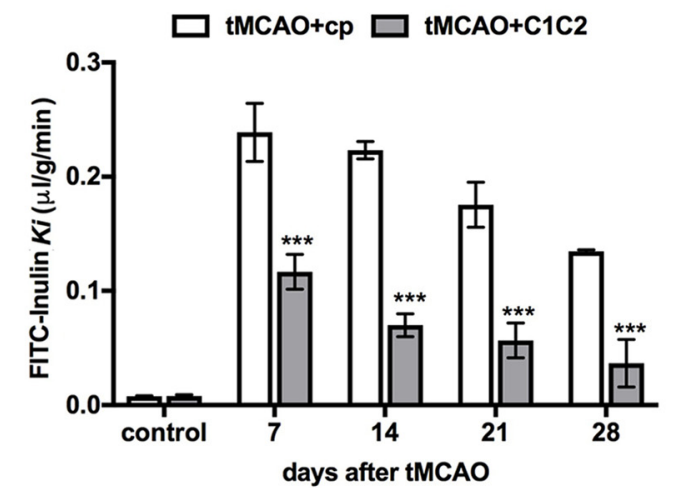

G claudin-5

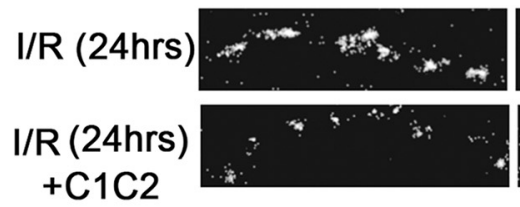

-3.

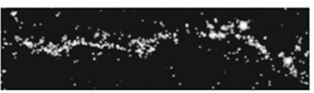

cldn $1^{\text {over }}+\mathrm{C} 1 \mathrm{C} 2$
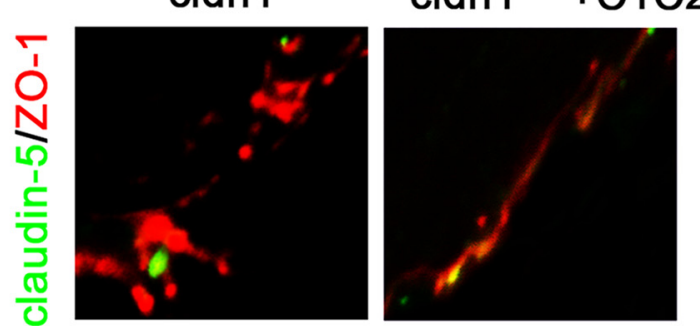

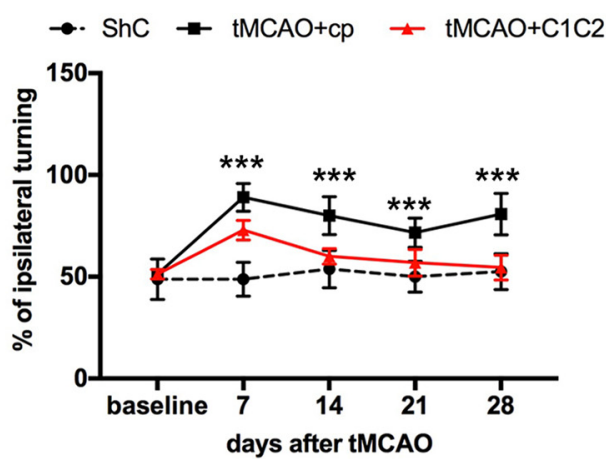

Figure 7. Claudin- 1 inhibition protects barrier integrity in chronic I/R conditions. A, Depletion of claudin- 1 with the claudin-1 peptide (C1C2; $1 \mu \mathrm{g} / \mathrm{ml}$; for specificity of C1C2 peptide, see Figure 7-1C, available at https://doi.org/10.1523/JNEUROSCI.1432-18.2018.f7-1) had no effect on barrier permeability to inulin in control mBMEC monolayers, but it decreased permeability in claudin1-overexpressing (cldn $1^{\text {over }}$ ) BMEC monolayers. Data are shown as means \pm SD. $n=3 ;{ }^{* * *} p<0.001$ control versus $\mathrm{cldn} 1^{\text {over }}\left(p=0.9998,{ }^{* * *} p=0.00074, p=0.462\right.$, ANOVA Tukey's post test) and $^{\# \#} p=0.00267 \mathrm{cldn} 1^{\text {over }}$ versus cldn $1^{\text {over }}+$ C1C2 (ANOVA, Tukey's post test) Time- and dose-dependent alteration in brain endothelial barrier permeability of cldn $1^{\text {over }}$. (Figure legend continues.) 


\section{Discussion}

Stroke induces BBB hyperpermeability, the magnitude of which varies temporally. Acutely, there can be an uncontrolled increase with extravasation of plasma proteins, leukocyte diapedesis, and vasogenic edema. However, subacutely and chronically, there may be smaller leaks that may lead to hemorrhagic transformation or, if they persist, excessive build-up of fluid leading to brain dysfunction, progression to chronic inflammation, and formation of microthrombi (Strbian et al., 2008; Kaur et al., 2011). The present study examined the cause of BBB leakage in the subacute and chronic phases of stroke, focusing on TJ complex alterations. We found that: (1) long-lasting and persistent BBB leakage after stroke is associated with TJ complex disorganization; (2) most leaky vessels expressed a "new" BBB claudin, claudin-1; (3) claudin-1 incorporates into the TJ complex via direct $\mathrm{ZO}-1$ interaction and by establishing homophilic cis- and trans-interactions; (4) the presence of claudin-1 is directly associated with decreased levels of claudin-5 trans-interaction and claudin-5/ ZO-1 interaction, and (5) claudin-1 may be a therapeutic target for reducing BBB permeability chronically after stroke and consequently improving neurological recovery. Blocking claudin-1 with a peptide had beneficial effects on BBB permeability and endothelial cell inflammatory phenotype.

In vitro and in vivo mouse and human studies all indicated an upregulation of a novel BBB claudin, claudin-1, in ischemic conditions. Claudins, major occlusive proteins, show cell specificity in expression and interactions. At the brain endothelium, the major claudin is claudin-5, but there are conditions (e.g., vasculogenesis, tumors, and traumatic brain injury) in which other claudins such as claudin-1 are present and might participate in paracellular space occlusion (Liebner et al., 2000; Shin et al., 2008). The cause and effects of claudin-1 expression are still unknown. Current hypotheses suggest an equal and beneficial role in BBB TJ complex organization, helping to seal the paracellular

\footnotetext{
$\leftarrow$

(Figure legend continued.) For BMEC monolayers treated with C1C2, see Figure 7-1A (available at https://doi.org/10.1523/JNEUROSCI.1432-18.2018.f7-1 and 7-1B). B, C1C2 treatment also reduced the inulin hyperpermeability induced by $\mathrm{I} / \mathrm{R}$ injury at 24 and $48 \mathrm{~h}$. Data are shown as means \pm SD. $n=3{ }^{* * *} p=0.000001,{ }^{* *} p=0.0015,0.0031,0.0028$ compared with control cells (ANOVA, Sidak's post test) and ${ }^{\# \# \#} p=0.000001,{ }^{\#} p=0.0265$ comparing the barrier permeability between cells with/without depletion of claudin-1 by C1C2 (ANOVA Sidak's post test). C, Superresolution images of TJ-associated claudin- 1 and claudin- 5 fragments on the cell border in cldn $1{ }^{\text {over }}$ BMECs, cldn $1^{\text {over }}$ BMECs treated with C1C2 claudin-1 peptide and I/R, and I/R BMEC monolayers treated with C1C2 claudin-1 peptide. C1C2 (1 $\mu \mathrm{g} / \mathrm{ml})$ treatment displaced claudin-1 from the cell-cell border and restored claudin-5 to the cell border. $\boldsymbol{D}$, Immunofluorescence staining for Z0-1, claudin-5, and/or claudin-1 in cldn $1^{\text {over }}$ BMEC, Cldn $1^{\text {over }}$ BMECs treated with C $1 C 2$ claudin- 1 peptide and I/R, and I/R BMEC monolayers treated with C1C2 claudin-1 peptide. C1C2 reduced colocalization of claudin-1 with Z0-1 and increased claudin-5/Z0-1 colocalization. $\boldsymbol{E}$, Protein array for proinflammatory mediators present in con-

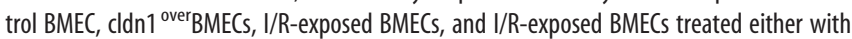
cldn1 shRNA or C1C2 peptide. Data represent several highly increased cytokines/chemokines in cell culture media collected at $24 \mathrm{~h}$. Depletion of claudin-1 by C1C2 peptide or cldn 1 shRNA decreased the expression of proinflammatory cytokines/chemokines. Data are shown as mean \pm SD. $n=3 .{ }^{* * *} p=0.000001$ compared with control (ANOVA Tukey's post test) and ${ }^{\# \# \#} p=0.000001$ (Tukey's test) compared with I/R-exposed cells. $\boldsymbol{F}, K_{\mathrm{i}}$ for FITC-inulin (5 kDa) in ischemic hemispheres at day 7-28 after transient MCA0. ShC, Sham control; tMCA0 +cp = treatment with control peptide (cp), tMCAO $+\mathrm{C} 1 \mathrm{C} 2$, treatment with claudin- $1 \mathrm{C} 1 \mathrm{C} 2$ peptide $(5$ $\mu \mathrm{g} / \mathrm{kg}$, i.p.). Data are shown as means \pm SD. $n=8 ;{ }^{* * *} p<0.001$ comparing $\mathrm{tMCAO}+\mathrm{cp}$ and tMCA0 + C1C2 (control- $p=0.999 ; 0.000084,0.000068,0.000062,0.000081$ for 7, 14, 21, $28 \mathrm{~d}_{\text {, }}$ ANOVA Tukey's post test). G, Corner turn test at days 1, 7, 14, 21, and 28 after tMCA0 in mice treated with control peptide (cp) and claudin-1 C1C2 peptide (5 $\mu \mathrm{g} / \mathrm{kg}$, i.p.). Data are shown as mean \pm SD. $n=8$ each group. ${ }^{* * *} p=0.00025, p=0.000001,0.000207$, and 0.000001 . ANOVA, Holm-Sidak's post test comparing tMCAO + cp and tMCAO+C1C2.
}

route, or via astrocytic expression, helping to form secondary astrocyte/glial barriers to protect the brain from endothelial breakdown (Liebner et al., 2000; Pfeiffer et al., 2011). These hypotheses are challenged by several controversial findings regarding the role of claudin-1 in TJ formation in BMEC: (1) claudin-1 mRNA expression is extremely low in resting BMECs compared with claudin-5 ( $\sim 3000$-fold less), pinpointing rather inducible features and roles in barrier regulation; (2) claudin-5 KO mice die just after birth with brain edema and hemorrhage, whereas claudin-1 KO mice die due to epidermal barrier and not BBB defects; and (3) BMEC claudin-1 mRNA and protein levels are not altered in claudin-5 KO mice, excluding a potential compensatory role (Nitta et al., 2003; Ohtsuki et al., 2007). This suggests that claudin-1 is not essential for BBB TJ complex function under physiological conditions and its appearance/induction is rather associated with altered TJ function. In addition, our results clearly suggest that claudin-1 appearance poststroke results in limited brain endothelial barrier recovery, partially due to restricted claudin-5 expression.

Acute stroke depletes many TJ proteins, forming a basis for BBB “opening” (Fernández-López et al., 2012). However, chronically, there is partial incomplete $\mathrm{BBB}$ recovery, with a pattern of increased TJ protein expression. In chronic stroke, BBB leakage might be due to insufficient TJ protein expression, which does not fully fit our results, or to a defect in TJ organization. Claudin-5 expression may be insufficient for establishing barrier properties due to slightly lower expression or incompatible interaction in the TJ complex. A potential compensatory claudin, claudin-12, showed increased expression, but due to an inability to build barriers, it is questionable how it affects barrier properties (Nitta et al., 2003). The appearance of claudin-1, a barriertype claudin, chronically in stroke raises the possibility that it may support and facilitate BBB recovery, reestablishing integrity, as indicated in a recent study on the beneficial effects of overexpressing claudin-1 in experimental autoimmune encephalomyelitis (Pfeiffer et al., 2011). It is important to note that significant claudin-1 overexpression is needed for barrier "rescue" (double transgenic claudin-1 mice), which, in our condition poststroke recovery, is potentially difficult to achieve.

Protein expression is a component establishing TJ properties. Equally important are TJ protein-protein interactions. Both claudin-1 and claudin-5 are "barrier type" claudins. Claudin cisand trans protein-protein interactions are important in TJ strand formation and paracellular occlusion (Piontek et al., 2008). Claudin-5 normally forms continuous strands with homotypic trans- and cis-interactions that are essential for oligomerization and creation of an impermeable TJ complex at the BMEC (Piontek et al., 2008; Rossa et al., 2014). Until recently, claudin-5 cisand trans-interactions at the BBB were described as homotypic. However, a new view of TJ complex organization suggests that heterogeneous claudin molecules constitute the backbone of TJ strands with heterophilic trans-interactions (Furuse et al., 1999; Milatz et al., 2015). In epithelial cells, claudin/claudin interactions have been defined as compatible (e.g., claudin-3 and claudin-5) or incompatible (e.g., claudin-1 and claudin-5), affecting TJ complex function in resting and injury conditions (e.g., claudin-1/claudin-5 interaction in lung injury) (Koval, 2013). In determining the potential effect of claudin- 1 on the BBB, our data indicated that claudin-1 generated intercellular strands based on homophilic claudin-1/claudin-1 cis- and trans- interactions without interaction with claudin-5. In chronic stroke conditions, claudin-5 strands are interrupted with claudin-1/claudin-1 strand fragments, making the barrier more a "piecemeal" struc- 
ture than continuous strands, contributing to barrier leakage. Claudin-1 influence also affects claudin-5/ZO-1 interaction. Claudin-5 requires ZO-1 interaction to localize on the cell membrane and establish trans-interactions between cells (Furuse et al., 1999). High expression of claudin-1 and its ability to accumulate on the membrane indicates that claudin- 1 and claudin-5 may compete for ZO-1 interaction, displacing claudin-5 from the TJ complex. Inhibition assays both with claudin-1 siRNA and claudin-1 peptide support this observation, showing that claudin-1 can be removed from the membrane and dissociated from ZO-1, increasing claudin-5 strand formation. Therefore, in the absence of claudin-5, claudin-1 might support sealing of the barrier by building its own strands, but it could be a limiting factor for full barrier recovery in cells with claudin- 5 due to their incompatible interactions.

It is important to highlight that this barrier destabilizing effect of claudin-1 is restricted to BMECs. Astrocytic claudin-1 expression has been reported, particularly in pathological conditions with BBB breakdown (Horng et al., 2017). A tight interaction between astrocytes potentially forms a secondary defense barrier against ischemic injury (Duffy et al., 2000; Horng et al., 2017). This protective ability of claudin-1 most probably occurs during acute injury. Therefore, the absence of astrocytic claudin-1 expression in chronic stroke could be the result of an already partial $\mathrm{BBB}$ recovery.

Alterations in the endothelial microenvironment or endothelial cell injury may induce claudin-1 chronically after stroke. That phase after stroke is closely associated with persistent low-grade cytokine and chemokine expression that affects BBB phenotype (Elahy et al., 2015; von Leden et al., 2017), potentially altering TJ protein expression (claudin-1 upregulation and claudin-5 downregulation). However, cell injury induces claudin-1 in diabetic nephropathy, alcoholic lung injury, and brain endothelial cell ischemia in vitro (Hasegawa et al., 2013; Schlingmann et al., 2016). In such conditions, claudin-1 upregulation mostly results from injury-induced epigenetic alterations driven by Sirt1 or miR155 (Hasegawa et al., 2013; Lopez-Ramirez et al., 2014). Therefore, claudin-1 induction may be a consequence of the cell injury and alterations in the microenvironment found in chronic poststroke conditions. Future study studies should clarify the signaling mechanisms of BBB injury in chronic stroke.

Could claudin-1 be an important new target for treating of $\mathrm{BBB}$ poststroke injury? Evidence suggests that persistent $\mathrm{BBB}$ leakage has a damaging rather than beneficial effects on poststroke recovery (Taheri et al., 2011) with build-up of perivascular edema and blood component extravasation leading to progressive chronic inflammation and formation of microthrombi (Krueger et al., 2015). Claudin-1 inhibition may affect BBB leakage, preventing these processes worsening BBB injury. Because claudin-1 expression also increases proinflammatory cytokine/ chemokine expression in BMECs, its "damaging" effect is not solely structural. Therefore, claudin-1 has good potential to fulfill the role of target for $\mathrm{BBB}$ recovery: it shows good translational properties and the same expression pattern poststroke in human and mice, it regulates barrier properties and endothelial phenotype, and as a surface structural protein it is accessible and can be removed by a small molecule strategy targeting endocytosis. This could bring benefits with low off-target effects.

Our results show the beneficial effects of the claudin-1 peptide C1C2 and claudin-1 shRNA in treating BBB hyperpermeability. Through high-affinity binding to the first extracellular loop of claudin-1, C1C2 diminishes trans- and cis-claudin-1 interactions, reduces claudin-1/ZO-1 interaction, redistributing claudin-1 to the cytosol for degradation (Staat et al., 2015). These effects result in continuous claudin-5 strands and decreased brain endothelial leakage. However, C1C2 has low affinity for claudin-5 and does not reduce its expression. By effecting both claudin-1 and claudin-5, C1C2 peptide activity is linked to increased permeability in barriers formed by multiple cell types (Staat et al., 2015). Care is needed in terms of both the dose and duration of C1C2 exposure if it is to be used as a therapy.

In conclusion, ischemia induces endothelial claudin-1 expression, where it plays a role in long-term poststroke BBB leakiness. Claudin-1 is a novel therapeutic target and reducing its expression may be important for preventing stroke recurrence and possibly poststroke brain injury.

\section{References}

Abbott NJ, Patabendige AA, Dolman DE, Yusof SR, Begley DJ (2010) Structure and function of the blood-brain barrier. Neurobiol Dis 37:13-25. CrossRef Medline

Abo-Ramadan U, Durukan A, Pitkonen M, Marinkovic I, Tatlisumak E, Pedrono E, Soinne L, Strbian D, Tatlisumak T (2009) Post-ischemic leakiness of the blood-brain barrier: a quantitative and systematic assessment by Patlak plots. Exp Neurol 219:328-333. CrossRef Medline

Chopp M, Zhang ZG, Jiang Q (2007) Neurogenesis, angiogenesis, and MRI indices of functional recovery from stroke. Stroke 38:827-831. CrossRef Medline

Deddens LH, Van Tilborg GA, Mulder WJ, De Vries HE, Dijkhuizen RM (2012) Imaging neuroinflammation after stroke: current status of cellular and molecular MRI strategies. Cerebrovasc Dis 33:392-402. CrossRef Medline

Dimitrijevic OB, Stamatovic SM, Keep RF, Andjelkovic AV (2006) Effects of the chemokine CCL2 on blood-brain barrier permeability during ischemia-reperfusion injury. J Cereb Blood Flow Metab 26:797-810. CrossRef Medline

Dirnagl U, Iadecola C, Moskowitz MA (1999) Pathobiology of ischaemic stroke: an integrated view. Trends Neurosci 22:391-397. CrossRef Medline

Duffy HS, John GR, Lee SC, Brosnan CF, Spray DC (2000) Reciprocal regulation of the junctional proteins claudin-1 and connexin43 by interleukin-1beta in primary human fetal astrocytes. J Neurosci 20: RC114. CrossRef Medline

Duncan PW, Lai SM, Keighley J (2000) Defining post-stroke recovery: implications for design and interpretation of drug trials. Neuropharmacology 39:835-841. CrossRef Medline

Durukan A, Marinkovic I, Strbian D, Pitkonen M, Pedrono E, Soinne L, Abo-Ramadan U, Tatlisumak T (2009) Post-ischemic blood-brain barrier leakage in rats: one-week follow-up by MRI. Brain Res 1280:158-165. CrossRef Medline

Elahy M, Jackaman C, Mamo JC, Lam V, Dhaliwal SS, Giles C, Nelson D, Takechi R (2015) Blood-brain barrier dysfunction developed during normal aging is associated with inflammation and loss of tight junctions but not with leukocyte recruitment. Immun Ageing 12:2. CrossRef Medline

Fernández-López D, Faustino J, Daneman R, Zhou L, Lee SY, Derugin N, Wendland MF, Vexler ZS (2012) Blood-brain barrier permeability is increased after acute adult stroke but not neonatal stroke in the rat. J Neurosci 32:9588-9600. CrossRef Medline

Fisher M, Schaebitz W (2000) An overview of acute stroke therapy: past, present, and future. Arch Intern Med 160:3196-3206. CrossRef Medline

Furuse M, Sasaki H, Tsukita S (1999) Manner of interaction of heterogeneous claudin species within and between tight junction strands. J Cell Biol 147:891-903. CrossRef Medline

Hasegawa K, Wakino S, Simic P, Sakamaki Y, Minakuchi H, Fujimura K, Hosoya K, Komatsu M, Kaneko Y, Kanda T, Kubota E, Tokuyama H, Hayashi K, Guarente L, Itoh H (2013) Renal tubular Sirtl attenuates diabetic albuminuria by epigenetically suppressing claudin-1 overexpression in podocytes. Nat Med 19:1496-1504. CrossRef Medline

Hawkins BT, Davis TP (2005) The blood-brain barrier/neurovascular unit in health and disease. Pharmacol Rev 57:173-185. CrossRef Medline

Horng S, Therattil A, Moyon S, Gordon A, Kim K, Argaw AT, Hara Y, Mariani JN, Sawai S, Flodby P, Crandall ED, Borok Z, Sofroniew MV, Chapouly C, 
John GR (2017) Astrocytic tight junctions control inflammatory CNS lesion pathogenesis. J Clin Invest 127:3136-3151. CrossRef Medline

Kaur J, Tuor UI, Zhao Z, Barber PA (2011) Quantitative MRI reveals the elderly ischemic brain is susceptible to increased early blood-brain barrier permeability following tissue plasminogen activator related to claudin 5 and occludin disassembly. J Cereb Blood Flow Metab 31:1874-1885. CrossRef Medline

Kazakoff PW, McGuire TR, Hoie EB, Cano M, Iversen PL (1995) An in vitro model for endothelial permeability: assessment of monolayer integrity. In Vitro Cell Dev Biol Anim 31:846-852. CrossRef Medline

Koval M (2013) Claudin heterogeneity and control of lung tight junctions. Annual review of physiology 75:551-567. CrossRef Medline

Krueger M, Bechmann I, Immig K, Reichenbach A, Härtig W, Michalski D (2015) Blood-brain barrier breakdown involves four distinct stages of vascular damage in various models of experimental focal cerebral ischemia. J Cereb Blood Flow Metab 35:292-303. CrossRef Medline

Liebner S, Fischmann A, Rascher G, Duffner F, Grote EH, Kalbacher H, Wolburg H (2000) Claudin-1 and claudin-5 expression and tight junction morphology are altered in blood vessels of human glioblastoma multiforme. Acta Neuropathol 100:323-331. CrossRef Medline

Lopez-Ramirez MA, Wu D, Pryce G, Simpson JE, Reijerkerk A, King-Robson J, Kay O, de Vries HE, Hirst MC, Sharrack B, Baker D, Male DK, Michael GJ, Romero IA (2014) MicroRNA-155 negatively affects blood-brain barrier function during neuroinflammation. FASEB J 28:2551-2565. CrossRef Medline

Milatz S, Piontek J, Schulzke JD, Blasig IE, Fromm M, Günzel D (2015) Probing the cis-arrangement of prototype tight junction proteins claudin-1 and claudin-3. Biochem J 468:449-458. CrossRef Medline

Nitta T, Hata M, Gotoh S, Seo Y, Sasaki H, Hashimoto N, Furuse M, Tsukita S (2003) Size-selective loosening of the blood-brain barrier in claudin5-deficient mice. J Cell Biol 161:653-660. CrossRef Medline

Ohno K, Chiueh CC, Burns EM, Pettigrew KD, Rapoport SI (1980) Cerebrovascular integrity in protein-deprived rats. Brain Res Bull 5:251-255. CrossRef Medline

Ohtsuki S, Sato S, Yamaguchi H, Kamoi M, Asashima T, Terasaki T (2007) Exogenous expression of claudin-5 induces barrier properties in cultured rat brain capillary endothelial cells. J Cell Physiol 210:81-86. CrossRef Medline

Pfeiffer F, Schäfer J, Lyck R, Makrides V, Brunner S, Schaeren-Wiemers N, Deutsch U, Engelhardt B (2011) Claudin-1 induced sealing of bloodbrain barrier tight junctions ameliorates chronic experimental autoimmune encephalomyelitis. Acta Neuropathol 122:601-614. CrossRef Medline

Piontek J, Winkler L, Wolburg H, Müller SL, Zuleger N, Piehl C, Wiesner B, Krause G, Blasig IE (2008) Formation of tight junction: determinants of homophilic interaction between classic claudins. FASEB J 22:146-158. CrossRef Medline

Rossa J, Protze J, Kern C, Piontek A, Günzel D, Krause G, Piontek J (2014) Molecular and structural transmembrane determinants critical for embedding claudin- 5 into tight junctions reveal a distinct four-helix bundle arrangement. Biochem J 464:49-60. CrossRef Medline

Schlingmann B, Overgaard CE, Molina SA, Lynn KS, Mitchell LA, Dorsainvil White S, Mattheyses AL, Guidot DM, Capaldo CT, Koval M (2016) Regulation of claudin/zonula occludens-1 complexes by hetero-claudin interactions. Nat Commun 7:12276. CrossRef Medline

Shin JS, Hyun SY, Kim DH, Lee S, Jung JW, Choi JW, Ko KH, Kim JM, Ryu JH (2008) Chronic hypoperfusion increases claudin-3 immunoreactivity in rat brain. Neurosci Lett 445:144-148. CrossRef Medline

Sladojevic N, Stamatovic SM, Keep RF, Grailer JJ, Sarma JV, Ward PA, Andjelkovic AV (2014) Inhibition of junctional adhesion molecule-A/LFA interaction attenuates leukocyte trafficking and inflammation in brain ischemia/reperfusion injury. Neurobiol Dis 67:57-70. CrossRef Medline

Staat C, Coisne C, Dabrowski S, Stamatovic SM, Andjelkovic AV, Wolburg H, Engelhardt B, Blasig IE (2015) Mode of action of claudin peptidomimetics in the transient opening of cellular tight junction barriers. Biomaterials 54:9-20. CrossRef Medline

Stamatovic SM, Johnson AM, Keep RF, Andjelkovic AV (2016) Junctional proteins of the blood-brain barrier: New insights into function and dysfunction. Tissue Barriers 4:e1154641. CrossRef Medline

Strbian D, Durukan A, Pitkonen M, Marinkovic I, Tatlisumak E, Pedrono E, Abo-Ramadan U, Tatlisumak T (2008) The blood-brain barrier is continuously open for several weeks following transient focal cerebral ischemia. Neuroscience 153:175-181. CrossRef Medline

Taheri S, Gasparovic C, Huisa BN, Adair JC, Edmonds E, Prestopnik J, Grossetete M, Shah NJ, Wills J, Qualls C, Rosenberg GA (2011) Blood-brain barrier permeability abnormalities in vascular cognitive impairment. Stroke 42:2158-2163. CrossRef Medline

Tietz S, Engelhardt B (2015) Brain barriers: crosstalk between complex tight junctions and adherens junctions. J Cell Biol 209:493-506. CrossRef Medline

von Leden RE, Khayrullina G, Moritz KE, Byrnes KR (2017) Age exacerbates microglial activation, oxidative stress, inflammatory and NOX2 gene expression, and delays functional recovery in a middle-aged rodent model of spinal cord injury. J Neuroinflammation 14:161. CrossRef Medline

Wong CH, Crack PJ (2008) Modulation of neuro-inflammation and vascular response by oxidative stress following cerebral ischemia-reperfusion injury. Curr Med Chem 15:1-14. CrossRef Medline

Yang Y, Rosenberg GA (2011) Blood-brain barrier breakdown in acute and chronic cerebrovascular disease. Stroke 42:3323-3328. CrossRef Medline 